Meta

Journal des traducteurs

Translators' Journal

\title{
La littérature québécoise traduite au Mexique : trois anthologies à la Foire internationale du livre de Guadalajara
}

\section{Madeleine Stratford et Louis Jolicoeur}

Volume 59, numéro 1, avril 2014

URI : https://id.erudit.org/iderudit/1026472ar

DOI : https://doi.org/10.7202/1026472ar

Aller au sommaire du numéro

\section{Éditeur(s)}

Les Presses de l’Université de Montréal

ISSN

0026-0452 (imprimé)

1492-1421 (numérique)

Découvrir la revue

Citer cet article

Stratford, M. \& Jolicoeur, L. (2014). La littérature québécoise traduite au Mexique : trois anthologies à la Foire internationale du livre de Guadalajara. Meta, 59(1), 97-123. https://doi.org/10.7202/1026472ar
Résumé de l'article

Le Québec était l'invité d'honneur de la Foire internationale du livre de Guadalajara tenue au cours de l'année 2003. Trois anthologies de littérature québécoise paraissent alors au Fondo de Cultura Económica mexicain : Latinos del norte, qui propose un tour d'horizon des principales tendances de la poésie québécoise de 1930 à 1960 ; El hilo de la memoria, qui présente vingt-quatre essayistes, nés entre 1920 et 1958, représentatifs du genre au Québec ; ¿ Un continente a la deriva ?, qui réunit les textes de seize nouvellistes contemporains, nés pour la plupart dans les années 1940 ou 1950. D’emblée, les trois publications semblent ainsi établir un parallèle entre le passé littéraire québécois et la poésie, entre le présent littéraire et la prose. Nous nous pencherons sur la façon dont ces trois livres présentent la littérature et la culture québécoises au lectorat mexicain et montrerons que le paratexte des ouvrages invite à les considérer comme complémentaires. Après avoir cerné leurs contextes de publication, de diffusion et de réception, nous les envisagerons d'un point de vue global en nous attardant aux éléments paratextuels (péritexte et épitexte) qui les distinguent : jaquettes, titres, préfaces, sélection des auteurs et des textes et discours ayant circulé dans la presse écrite.
Tous droits réservés @ Les Presses de l’Université de Montréal, 2014
Ce document est protégé par la loi sur le droit d'auteur. L'utilisation des services d'Érudit (y compris la reproduction) est assujettie à sa politique d'utilisation que vous pouvez consulter en ligne.

https://apropos.erudit.org/fr/usagers/politique-dutilisation/ 


\title{
La littérature québécoise traduite au Mexique: trois anthologies à la Foire internationale du livre de Guadalajara*
}

\author{
MADELEINE STRATFORD \\ Université du Québec en Outaouais, Gatineau, Canada \\ madeleine.stratford@uqo.ca
}

LOUIS JOLICOEUR

Université Laval, Québec, Canada

louis.jolicoeur@lli.ulaval.ca

\begin{abstract}
RÉSUMÉ
Le Québec était l'invité d'honneur de la Foire internationale du livre de Guadalajara tenue au cours de l'année 2003. Trois anthologies de littérature québécoise paraissent alors au Fondo de Cultura Económica mexicain: Latinos del norte, qui propose un tour d'horizon des principales tendances de la poésie québécoise de 1930 à 1960; El hilo de la memoria, qui présente vingt-quatre essayistes, nés entre 1920 et 1958, représentatifs du genre au Québec; ¿ Un continente a la deriva?, qui réunit les textes de seize nouvellistes contemporains, nés pour la plupart dans les années 1940 ou 1950. D'emblée, les trois publications semblent ainsi établir un parallèle entre le passé littéraire québécois et la poésie, entre le présent littéraire et la prose. Nous nous pencherons sur la façon dont ces trois livres présentent la littérature et la culture québécoises au lectorat mexicain et montrerons que le paratexte des ouvrages invite à les considérer comme complémentaires. Après avoir cerné leurs contextes de publication, de diffusion et de réception, nous les envisagerons d'un point de vue global en nous attardant aux éléments paratextuels (péritexte et épitexte) qui les distinguent: jaquettes, titres, préfaces, sélection des auteurs et des textes et discours ayant circulé dans la presse écrite.
\end{abstract}

\section{ABSTRACT}

Quebec was featured as guest of honour at the 2003 Guadalajara International Book Fair. On that occasion, three anthologies of Quebec literature were published by the Fondo de Cultura Económica: Latinos del norte gives an overview of the main tendencies in Quebec poetry from 1930 to 1960; El hilo de la memoria introduces 24 essay writers born between 1920 and 1958 who represent this genre in Quebec; ¿Un continente a la deriva? collects the short stories of 16 contemporary Quebec writers born for the most part in the 1940s or 1950s. At first glance, the three books seem to be drawing a parallel between Quebec's literary past and poetry, between its literary present and prose. The aim of this article is to examine how the three anthologies present Quebec literature and culture to Mexican readers. The publications share enough paratextual similarities to be seen as complementing each other. After outlining the publication, distribution and reception contexts of the books, the authors analyse key paratextual elements from a global viewpoint: book jackets; titles; prefaces; author and text selection; comments in the press.

\section{MOTS-CLÉS/KEYWORDS}

littérature québécoise, Mexique, paratexte, paratraduction, diffusion Quebec literature, Mexico, paratext, paratranslation, circulation 


\section{Introduction}

La Foire internationale du livre (Feria Internacional del Libro; FIL) de Guadalajara, qui est le plus grand salon du livre dans le monde hispanique, choisissait en 2003 le Québec comme invité d'honneur. Une délégation d'une cinquantaine de personnes s'y est rendue: auteurs, éditeurs, traducteurs, journalistes, universitaires, chanteurs, danseurs et artistes variés. La délégation était impressionnante et révélait sans l'ombre d'un doute que le Québec voulait profiter de l'occasion au maximum, mais de façon efficace et à un coût raisonnable, surtout après le succès mitigé de la Saison du Québec à Paris en 1999 et l'échec de la Saison du Québec à New York (lancée quelques jours avant le 11 septembre 2001, puis annulée après les attaques sur les tours jumelles). Avant le Québec, le Canada avait été l'invité d'honneur de la Foire de Guadalajara, en 1996, mais de l'avis de l'Union des écrivaines et écrivains du Québec (UNEQ) et d'autres intervenants du milieu littéraire québécois, le Québec n’avait pas su tirer profit de cette tribune consacrée avant tout au Canada: c'est surtout le pays qui a fait «bonne figure» à cette occasion "grâce à la locomotive québécoise», et non l'inverse (Morissette 1996)1 ${ }^{1}$ Les choses allaient être différentes à Guadalajara. Plutôt que des coups d'éclat, qui risquent de se convertir rapidement en coûteux coups d'épée dans l'eau, on allait d'abord penser aux livres.

Pour près de la moitié des trente-huit auteurs invités à Guadalajara à l'occasion de la FIL (annexe 1), le kiosque du Québec proposait sur place des traductions en espagnol - souvent faites au Mexique, mais pas exclusivement. Certains ouvrages avaient aussi été préparés spécialement pour l'événement, dont trois anthologies de littérature québécoise, parues au prestigieux Fondo de Cultura Económica (FCE)², qui représentaient, respectivement, les genres de la nouvelle, de la poésie et de l'essai: ¿Un continente a la deriva? Antología de narradores de Quebec, présentée et préfacée par Gilles Pellerin (2003a) $)^{3}$ Latinos del norte. Antología de poesía de Quebec, présentée et préfacée par Bernard Pozier (2003a) ${ }^{4}$, et El hilo de la memoria. Antología de ensayo de Quebec, présentée et préfacée par Jean-François Chassay (2003a). Financé par l'État mexicain, le FCE est l'une des principales maisons d'édition dans ce pays, voire en Amérique latine: "El FCE es la editorial de México y de Iberoamérica», peut-on lire dans la page de son site Internet faisant état de son histoire (nous soulignons) ${ }^{6}$. Déjà, l'inclusion des trois anthologies dans la collection Tierra Firme constitue en quelque sorte une prise de position de la part des éditeurs. En effet, ils semblent, par ce geste, considérer la littérature québécoise comme faisant partie de la culture latino-américaine, cette collection ayant été lancée dans le but de présenter les œuvres d'origine et de thématique latino-américaines?

Étant donné la notoriété du FCE au Mexique, il n'y a pas lieu de s'étonner que les trois ouvrages aient bénéficié d'une couverture appréciable dans la presse écrite comparativement aux autres livres traduits à l'occasion de la FIL. Dans le dossier de presse préparé par le Bureau des événements du Québec 8 , 18 articles traitent de l'une ou l'autre des anthologies (annexe 2). De plus, le dossier comprend un supplément publicitaire ${ }^{9}$ et quelques annonces ${ }^{10}$ visant à promouvoir toutes les anthologies lancées à la FIL par le FCE ou seulement Latinos del norte. Plusieurs journalistes mentionnent une ou deux des anthologies, mais la majorité (8 sur 18) présentent les trois anthologies ensemble comme s'il s'agissait d'une trilogie: « reúnen a medio centenar de voces representativas de la creación literaria quebequense en los généros de poesía, narrativa y ensayo", explique-t-on dans El Universal (Anonyme 29 novembre 2003). 
En 2003, la presse mexicaine a même surnommé «paquete Quebec» (Ortiz Partida $1^{\text {er }}$ décembre 2003a) l'ensemble de neuf ouvrages lancés par le FCE spécifiquement pour la FIL. On peut lire, encore dans le quotidien El Universal, que ces nouveautés du FCE constituent en fait «el paquete editorial más abarcador y rico [...] que en su conjunto brinda un panorama plural de la literatura y la cultura de Quebec» (Anonyme 29 novembre 2003). Dans les médias québécois, Caroline Montpetit, du Devoir, annonce dès décembre 2002 la préparation des trois anthologies (Montpetit 9 décembre 2002), et en reparle quelques mois plus tard (Montpetit 6 mars 2003), ce qui renforce l'impression qu'il s'agit de «triplés». Les trois livres partagent assez de similitudes pour que l'on soit tenté de les traiter en bloc: ils ont tous trois reçu l'appui financier du Gouvernement du Québec, ont été tirés à 2000 exemplaires, et sont le fruit d'une collaboration avec une maison d'édition québécoise: L'instant même pour ¿Un continente a la deriva?, Écrits des Forges pour Latinos del norte, et les Éditions du Boréal pour El hilo de la memoria ${ }^{11}$. Sur le plan du contenu, l'anthologie d'essais se démarque cependant des deux autres, l'essai étant un genre souvent perçu comme non proprement «littéraire» par le grand public, pour qui les genres littéraires se limitent souvent au roman (et à la nouvelle), à la poésie et au théâtre. Chassay reconnaît le caractère «hybride» de l'essai et insiste sur le fait qu'il possède une qualité «littéraire» au même titre que le roman, le théâtre et la poésie (Chassay, cité dans Ortiz Partida 2 décembre 2003; voir annexe 2). D’ailleurs, la nature «littéraire» de l'essai fait aussi l'objet de débats parmi les chercheurs (à cet égard, voir, notamment, Audet [2002]).

Nous désirons ici nous pencher plus avant sur la façon dont les trois anthologies ont présenté la littérature et la culture québécoises au lectorat mexicain. Nous nous intéresserons notamment à l'image du Québec et de sa littérature projetée dans le paratexte des ouvrages. Comme l'explique Gérard Genette (1987: 7), le paratexte a une influence sur la réception d'un ouvrage, car il sert non seulement à "présenter" le texte en ceci qu'il le fait connaître des lecteurs, mais il se porte aussi garant de la «présence» physique du texte, car c'est lui qui lui donne une forme tangible. En se basant sur l'analyse du paratexte d'ouvrage traduit, cette étude se situe dans le cadre du concept de paratraduction élaboré par José Yuste Frías à l'Université de Vigo, qui vise à rendre à l'image et à tout aspect visuel de la traduction et de ses paratextes l'importance qu'ils revêtent dans la construction de la signifiance d'une ouvre traduite. Adaptant les propos de Genette, Yuste Frías explique qu'il n'existe pas de traduction sans paratraduction: «Le texte traduit [...] n'a de fin que lorsqu'il est [...] accompagné, [...] et présenté par un ensemble de productions paratextuelles traduit» (Yuste Frías 2010: 311).

Nous basant sur les propos de Genette et de Yuste Frías, et tenant pour acquis qu'avant même d'avoir lu un livre, un lecteur potentiel s'est vraisemblablement déjà fait une idée de son contenu à partir de l'effet que son paratexte aura eu sur lui, nous étudierons le péritexte éditorial (jaquettes, titres, sélection d'auteurs tels que présentés dans la table des matières et préfaces) des trois anthologies. Nous considérerons aussi l'épitexte des ouvrages, c'est-à-dire les discours qui ont circulé à leur sujet dans les articles de journaux mexicains, répertoriés dans leur presque totalité dans le dossier de presse du Bureau des événements du Québec, deux cédéroms qui contiennent quelque 1055 articles et publicités parus de la mi-août 2001 à la mi-janvier 2004 dans des quotidiens, des hebdomadaires ainsi que des magazines, au Mexique, 
au Québec et dans le monde hispanique. Cette analyse comparée du paratexte des trois livres permettra d'évaluer qualitativement la façon dont la littérature québécoise de langue française a été présentée aux Mexicains en 2003 par le FCE.

\section{Les pages de couverture : un hommage à Saint-Denys Garneau}

La couverture est le premier élément perçu par toutes les personnes susceptibles d'entrer en contact avec un livre, qu'ils en soient ou non les lecteurs. En particulier, la première de couverture aura un impact sur la diffusion de l'ouvrage, ou du moins sur la perception que le public en aura. Ici, les couvertures des trois anthologies présentent une mise en page similaire. D'emblée, on peut penser que le parallélisme de la forme est dû au fait que celles-ci appartiennent à une même collection, Tierra Firme. Mais cela n'est pas tout: la page comportant le copyright et le numéro d'ISBN indique également, pour chacune, que la couverture est ornée d'une peinture réalisée dans les années 1930 par le poète Hector de Saint-Denys Garneau. Les images des anthologies de poèmes et d'essais sont tirées du catalogue d'exposition L'Univers de Saint-Denys Garneau paru aux Éditions du Boréal en collaboration avec le Musée d'art de Joliette (Gascon 2011) ${ }^{12}$, tandis que la toile illustrant les nouvelles provient de la collection privée de Giselle Huot. Or, même si Garneau se voyait autant peintre que poète ${ }^{13}$, c'est pour son œuvre poétique qu'on le connaît au Québec. Il s'agit d'ailleurs du premier poète traduit présenté par Pozier. Ce choix d'illustrations contribue donc à tisser un lien poétique subtil entre les trois livres.

Chaque illustration semble avoir été choisie spécifiquement pour le genre littéraire présenté, empreinte d'un symbolisme qui oriente déjà la lecture, et l'effet d'ensemble créé par les trois tableaux évoque plus le passé que le présent du Québec. Ainsi, la couverture de Latinos del norte est orné de La liseuse ${ }^{14}$, probablement la toile la plus connue de Garneau. Elle a donné son nom au Chemin de la Liseuse, piste cyclable qui longe la rivière Jacques-Cartier ${ }^{15}$, et illustre presque tous les rares articles de journaux consacrés à l'œuvre peint du poète ${ }^{16}$. La poésie étant souvent associée à une muse éthérée ${ }^{17}$, il est naturel sur le plan symbolique que ce soit une femme, qui plus est sans visage, qui représente ici la poésie. Cette «Liseuse à la robe rose», écrit François Hébert, semble «absorbée dans sa lecture au point que son visage a perdu ses traits» et son livre est mis en valeur «au centre de la toile» (Hébert 1998: 12-13). Cette insistance sur l'acte de la lecture - probablement de poèmes - a peut-être contribué à nourrir le bruit qui courait dans les journaux mexicains durant la FIL selon lequel la poésie serait le genre littéraire le plus lu des Québécois ${ }^{18}$.

Par ailleurs, le lien entre la peinture de Garneau et le titre de l'anthologie, Latinos del norte, mérite d'être souligné, car ceux-ci semblent entrer en dialogue. D’une part, c'est un être humain, plus qu'un paysage, que l'on voit ici. Comme le titre de l'anthologie, où Latinos forme le «noyau» du syntagme, l'image choisie met l'accent sur les habitants du Québec. Il en résulte que la poésie paraît intimement liée au peuple québécois, à son identité profonde. D’autre part, le paysage dans lequel Garneau a peint sa "liseuse» évoque une «latinité» plus qu'une "nordicité»: la femme lit au soleil, vêtue d'une robe d'été, agenouillée sur un sol jaune et sablonneux. Avec ses manches bouffantes et ses cheveux relevés en chignon, cette femme a presque l'air d'une Maria Chapdelaine que l'on aurait transplantée quelque part au Mexique. En somme, on dirait que le but est d'afficher ici le côté ensoleillé (latin?) du Québec. 
Pour sa part, le titre de l'anthologie de nouvelles, ¿Un continente a la deriva?, renvoie non pas aux gens, mais au territoire lui-même, au «continent» que l'on peut supposer «nord-américain» si l'on cherche à unir le Mexique et le Québec. Ici, l'huile de Garneau, La vieille chaufferie ${ }^{19}$, représente une chaumière pittoresque entre les arbres fournis d'un boisé. Cette toile, aux couleurs plus froides que la précédente, s'approche de l'image bucolique que l'on pourrait avoir du Québec d'antan, susceptible d'être associée à l'époque de Maria Chapdelaine. On pourrait s'imaginer la Liseuse habiter cette forêt verdoyante, mais cette vision du terroir détonne avec les propos de la quatrième de couverture, qui soulignent le caractère contemporain de la nouvelle québécoise. Chose sûre, l'effet créé diffère des grands espaces blancs à la Jean-Paul Lemieux, souvent utilisés pour représenter une certaine froideur, voire une certaine angoisse québécoise.

Par contraste avec les anthologies de Pozier et de Pellerin, c'est la ville, Montréal, et non la campagne, qui illustre l'anthologie de Chassay. Bien que cette Arrière-cour ${ }^{20}$ de Westmount soit toujours l'œuvre de Garneau, celle-ci diffère passablement des deux autres, en ceci que l'on n'y voit presque plus de verdure: seulement un peu de gazon jauni et un arbre dépouillé de ses feuilles. Si une nette impression de froid se dégage du ciel blanc et de l'arbre émacié, il n'y a cependant pas de neige au sol. Contrairement aux illustrations des deux autres anthologies, rien dans cette toile n'évoque le terroir québécois. Sur le plan symbolique, l'essai apparaît donc d'emblée comme un genre plus cérébral, plus moderne, moins romantique, voire moins proprement «littéraire» que la poésie et la nouvelle. Dans le titre, il n'est d'ailleurs plus question de l'habitant ou de son continent, mais de sa «mémoire», une mémoire aussi citadine que l'inscription «Je me souviens» sur les plaques d'immatriculation.

\section{Des sous-titres parlants et des titres intrigants}

Selon Genette, le titre d'un livre peut assumer, de manière générale, quatre fonctions communicatives: 1) identifier le livre; 2) indiquer ce dont il traite ou de quel type de livre il s'agit; 3) connoter le sujet du livre ou son contenu; 4) séduire (Genette 1987: 96-97). Les trois anthologies ont ceci en commun qu'elles portent toutes trois un sous-titre dénotatif caractéristique qui explique le type de livre présenté («antología»), le genre des textes sélectionnés («poesía», «narradores», "ensayo») et la provenance des auteurs («de Quebec»). Par contraste, les trois titres principaux, écrits en grosses lettres au centre de la couverture, sont de nature clairement connotative et semblent viser à séduire le public mexicain, ou du moins à piquer sa curiosité. Toutefois, nous verrons que les différents effets créés les distinguent.

Fait intéressant, sur la page comportant le copyright de Latinos del norte, une inscription indique que le titre original du livre aurait été Latins du nord. Or, le lecteur cherchera en vain ce soi-disant «original» français, car les Écrits des Forges n'ont jamais publié un ouvrage sous ce titre, ou contenant exactement la même sélection de poèmes. En fait, ce que la mention d'un titre «original» français porte à croire est que le titre de l'anthologie a peut-être d'abord été choisi en français, puis traduit en espagnol. Quoi qu'il en soit, comme l'anthologie de poèmes est une édition bilingue, le titre français (qu'il soit «original» ou non) renvoie de toute évidence au même livre que le titre espagnol. En revanche, bien qu'il n'y ait aucune allusion au «titre original» dans l'anthologie de Pellerin (2003a; voir note 3) ni dans celle de 
Chassay (2003a; voir note 5), toutes deux ont paru au Québec la même année (Pellerin 2003b; Chassay 2003b, voir notes 3 et 5) en édition unilingue française. Cela donne à penser que les deux projets ont été pensés essentiellement en fonction de la formidable tribune qu'offrait la FIL de Guadalajara, et en prévision de sa traduction immédiate, mais aussi dans un désir de présenter aux Québécois les auteurs de deux genres peut-être moins bien connus du grand public que la poésie. Notons cependant que les versions françaises et espagnoles ne sont pas parues tout à fait au même moment. Dans le cas de l'anthologie de nouvelles, Pellerin déclare «qu'aucune anthologie de la nouvelle n'était parue au Québec avant que les Mexicains en fassent la demande» (cité par Bellemare-Page 2004: 16), et la version française est donc parue après sa traduction espagnole, ce qui est pour le moins inhabituel. En revanche, l'anthologie d'essais a été publiée le 15 septembre 2003 selon Boréal (voir note 5), soit environ un mois avant la traduction espagnole. Par ailleurs, ce qui étonne, dans ces deux cas, c'est la nature résolument informative des titres français, par contraste avec les titres espagnols. En français, toute prétention de séduction a disparu: Anthologie de la nouvelle québécoise actuelle (Pellerin 2003b) et Anthologie de l'essai au Québec depuis la Révolution tranquille (Chassay 2003b). Cette sobriété des titres semble toutefois refléter une tendance du monde de l'édition au Québec. En effet, Jane Everett et Sophie Marcotte expliquent que «les titres des anthologies contemporaines sont généralement discrets quant à la dimension sélective de leur projet», et presque tous les exemples qu'elles donnent dans leur article sont essentiellement informatifs (Everett et Marcotte 2010: 8).

\subsection{Latinos del norte: un titre vendeur}

Selon Edgar Alejandro Hernández, Bernard Pozier aurait intitulé son anthologie Latinos del norte en raison des nombreux parallèles culturels entre le Mexique et le Québec, dont l'influence catholique, les langues latines et l'appartenance à l'Amérique du Nord (Pozier, cité par Hernández 2003). Selon Gaston Bellemare, ce seraient les Mexicains qui, constatant notre latinité commune, auraient eux-mêmes baptisé ainsi l'anthologie (Apostolska 14 mars 2011) ${ }^{21}$. Peu importe que le titre ait été choisi par Pozier lui-même ou par les éditeurs du FCE, il reste que celui-ci est lourd de symbolisme. Selon Claude Beausoleil (2004: 52), ce titre aurait «donné le ton à l'ensemble des commentaires» formulés dans la presse écrite.

En fait, il y a déjà longtemps que l'expression latinos del norte circule, au Mexique comme au Québec, en référence aux Québécois. Le traducteur Marco Antonio Campos raconte: «Lorsque, en 1987, nous avons organisé à Querétaro la Rencontre des poètes du monde latin, quelqu'un a dit que nous devrions inviter les Québécois, qui sont aussi latins ${ }^{22}$.» Selon la poète et traductrice mexicaine Silvia Pratt, l'écrivain Lazlo Moussong, en désignant la latinité comme point commun entre le Mexique et le Québec, aurait incité plus d'un Mexicain à assimiler les Québécois à des Latinos del norte au début des années 1980 (Pratt 2007 : 38). Selon Victor Armony, cependant, ce serait plutôt le sociologue québécois Marcel Rioux qui aurait lancé le concept en 1974, les politiciens québécois s'empressant ensuite de l'exploiter à leur avantage (Armony 2002: 22).

$S$ 'il est difficile de remonter à l'origine exacte de l'appellation, la récupération politique à laquelle Armony fait allusion est palpable dans les années précédant la 
FIL. Déjà en 1999, Bernard Landry avait utilisé l'expression en entrevue avec la presse mexicaine ${ }^{23}$. Deux ans plus tard, Landry, alors premier ministre, prononçait en avril 2001 une allocution intitulée Nous sommes les Latins du Nord à l'occasion du Sommet des Amériques. Le 20 mai 2002, Louise Beaudoin, ministre des Relations internationales, affirmait à Beverly Hills: "We are los Latinos del norte ${ }^{24}$ ». Le parallèle que le gouvernement Landry établit alors entre le Québec et le Mexique, et ce, tout au long de son mandat, ne pouvait que rejaillir sur l'image du Québec projetée lors de la FIL.

Par ailleurs, rappelons que la FIL de Guadalajara a d'abord été planifiée, du côté québécois, sous la gouverne de Bernard Landry. C'est son gouvernement qui en a supervisé les préparatifs jusqu'à la défaite du Parti québécois en avril 2003. Une certaine tension politique se fit d'ailleurs sentir à l'approche de la FIL. Par exemple, la journaliste Caroline Montpetit raconte que «l'ambassadeur canadien au Mexique aurait offert au Fondo de cultura económica, qui publie les anthologies [qui font l'objet du présent article], de lui acheter 500 exemplaires de ses livres si on inscrivait que cette littérature est canadienne plutôt que québécoise» (Montpetit 2003). Or, le FCE n'a visiblement pas accédé à cette demande, puisque les trois ouvrages se rattachent clairement à la littérature proprement québécoise, et remercient d'ailleurs ouvertement «el apoyo del gobierno de Quebec». Dans un tel contexte, il n'est pas étonnant que l'anthologie Latinos del norte, que l'on commença à préparer au moins une bonne année avant la FIL, porte un titre qui évoque le gouvernement péquiste de Landry.

Si nos deux cultures, effectivement latines, partagent des similitudes indéniables, un commentaire de la journaliste Miryam Audiffred (29 novembre 2003; voir annexe 2) incite à penser que cette insistance sur notre latinité commune pourrait aussi cacher, dans le contexte de la FIL, une certaine visée mercantile: «Encontrar lazos entre los universos literarios de México y Quebec implica, para los escritores de esa región, sentar las bases de una familiaridad susceptible de ganar la inclusión de la literatura quebequense en la literatura de América. » Bref, le titre de l'anthologie de poésie contribue à générer un sentiment d'appartenance sinon à une même culture, du moins à une même littérature latine, ce qui en fait un titre qui n’est pas nécessairement aussi politique qu'on pourrait le croire, mais en tous les cas résolument «vendeur».

\section{2. ¿Un continente a la deriva?: une question sans réponse}

Contrairement au titre de l'anthologie de poèmes, évocateur mais sans détour, revendicateur même, celui de l'anthologie de nouvelles demeure ambigu. Ce continent à la dérive évoque-t-il cette terre francophone en périphérie de l'Amérique, tant sur le plan géographique que culturel et linguistique? Sans doute. Mais la dérive n'évoquet-elle pas également une sorte de décadence, d'abandon, d'échec? Cela demeure difficile à valider cependant, dans le cadre du moins de l'aventure éditoriale en cause. La présentation du Québec qui y est faite, tant par le choix des auteurs de l'anthologie que par les thèmes traités par ceux-ci, ne prête guère le flanc à ce type d'interprétation. Aucune victimisation particulière dans ces textes, aucune dérive culturelle, politique ou autre qui soit particulièrement mise en relief.

Le critique José Luis Enciso lit le mot continente du titre au sens propre, l'associant au territoire (terroir?) québécois, mais aussi au figuré, comme métaphore du 
domaine de la nouvelle littéraire. Il opine que les textes sélectionnés répondent de deux façons à la question posée par le titre. Sur le plan de l'Histoire, un «continent» qui produit sa propre littérature, traduisant ainsi sa vision du monde, ne peut être considéré «à la dérive»; sur le plan de l'histoire, de la structure narrative qu'elle privilégie généralement, la nouvelle québécoise n’est pas «à la dérive» non plus: elle a une trame, dit-il, ne serait-ce que celle de «l'anecdote développée, mais diluée» (Enciso 29 novembre 2003).

Quoi qu'il en soit, ce titre peut difficilement avoir été choisi sans l'influence, voire l'intervention, ou du moins l'approbation du préfacier. La préface ne fait d'ailleurs référence à aucun abandon ni échec, mais le discours sur les cultures périphériques qu'on y retrouve pourrait sans doute donner quelques pistes quant aux interprétations à donner à ce titre aussi ambigu que poétique. Si, comme dans le cas de l'anthologie de poèmes, la préface et la sélection d'auteurs visent à présenter la littérature et la culture québécoises au lectorat mexicain, on voit en effet poindre un désir de rapprocher Mexicains et Québécois à travers une périphérie commune. C'est à se demander si le continent à la dérive évoqué dans le titre ne serait pas l'Amérique latine dans son ensemble, le Québec y compris, imaginé en train de se détacher du géant étatsunien, un peu comme le Portugal de Saramago s'éloignant peu à peu de l'Europe...

\subsection{El hilo de la memoria : la rhétorique de l'histoire}

Si les titres des deux premières anthologies appellent essentiellement à l'amitié, voire à la solidarité entre le Québec et le Mexique, c'est plutôt la métaphore de la mémoire, du souvenir, bref du fil (ténu par définition, fragile donc) entre passé et présent, qui semble se dessiner derrière le titre de l'anthologie d'essais. Bien que Chassay insiste sur l'actualité des thèmes évoqués par les essayistes (la vie quotidienne, la condition de la femme, la situation des immigrants, l'écologie, les luttes politiques), le titre, comme le confirme la préface (voir plus bas), met sans contredit l'accent sur l'importance des liens entre ces grandes questions d'aujourd'hui et le passé dont elles sont issues, tant sur le plan de l'histoire et de la géographie que des luttes politiques qui ont marqué le Canada et le Québec depuis le début de la colonie.

Outre ces thématiques, on perçoit également dans le titre de cette anthologie un clin d'œil esthétique ${ }^{25}$, par l'entremise de la métaphore, ce que vient corroborer la préface et le choix de textes, où la modernité (au sens formel et politique), la contemporanéité et le changement social sont tour à tour évoqués. Ce fil (d'Ariane?) que tissent les penseurs québécois entre le passé et l'époque moderne vient manifestement mettre en lumière la modernité du Québec autant que l'importance de son passé. Le fait que ces auteurs, contrairement à ceux des deux autres anthologies, aient tous plus de 40 ans, ce qu'évoque Chassay dans sa préface tout en le déplorant (Chassay $2003 c)^{26}$, pourrait par ailleurs bien confirmer qu'il convient de prendre un peu d'âge pour accéder à la modernité...

\section{Les préfaces : lieux de prises de position}

Une préface, comme le souligne Bourdieu (2002: 5), est un acte typique de transfert de capital symbolique. C'est un marquage, au même titre que le sont la jaquette, le sceau de l'éditeur, la collection, etc.: 
[...] le transfert d'un champ national à un autre se fait à travers une série d'opérations sociales: une opération de sélection (qu'est-ce qu'on traduit? qu'est-ce qu'on publie? qui traduit? qui publie?); une opération de marquage (d'un produit préalablement «dégriffé») à travers la maison d'édition, la collection, le traducteur et le préfacier [...]; une opération de lecture enfin, les lecteurs appliquant à l'œuvre des catégories de perception et des problématiques qui sont le produit d'un champ de production différent. (Bourdieu 2002: 5)

Les préfaces des anthologies de nouvelles, de poèmes et d'essais, comme la sélection des auteurs, comme il a été mentionné, sont le fait des directeurs des anthologies.

Ici, le fait que la préface de Latinos del norte rédigée par Pozier (Pozier 2003b) ${ }^{27}$ soit particulièrement partisane n'est pas sans répercussion sur l'image du Québec au Mexique. En effet, Pozier y établit le statut national de la province et souligne la dichotomie entre le peuple québécois et le bloc anglophone (Pozier 2003b: 7). Il y proclame sans hésiter que «la quasi-totalité des intellectuels et des artistes québécois sont indépendantistes, séparatistes ou en faveur de l'autonomie» (Pozier 2003b : 8; nous traduisons $)^{28}$. Selon lui, la poésie actuelle s'est donné pour tâche de propager le statut de nation autonome du Québec (Pozier 2003b : 8). Le portrait du Québécois du début du $\mathrm{xx}^{\mathrm{e}}$ siècle a tout pour émouvoir le lecteur mexicain et faire en sorte qu'il s'y identifie: «sans continent, parce que les États-Unis en ont usurpé le nom, sans pouvoir, car sous la férule des nantis anglophones, maintenu dans l'ignorance par son propre gouvernement et privé de plaisirs par la censure de son clergé» (Pozier 2003b: 10 ; nous traduisons) ${ }^{29}$. Couvrant les années 1960, Pozier décrit le mouvement nationaliste comme l'un des plus importants de l'histoire de la poésie québécoise. Là encore, sa vision indépendantiste s'exprime dans l'idée d'un Québec souverain né de l'imaginaire poétique pour être "peu à peu assumé sur le plan politique et économique» (Pozier 2003b: 11 ; nous traduisons) ${ }^{30}$. Enfin, lorsque Pozier s'attarde aux années 1980 et 1990, il les dit marquées par un désintéressement pour les grands mouvements collectifs et par un retour à l'introspection. Cela semble contredire l'idée selon laquelle la poésie actuelle serait essentiellement nationaliste. Pozier explique ce paradoxe en disant que toutes les générations de poètes sont éditées ou rééditées au cours des années 1990.

On constate la répercussion des propos de Pozier dans la presse mexicaine, où une majorité des articles traitant de l'une ou l'autre des anthologies (10 sur 18) font référence à l'indépendantisme ou au nationalisme québécois (voir annexe 2, colonne A). Dans six articles (voir annexe 2, colonne B), on rapporte directement ou indirectement ses paroles ou des extraits de sa préface. Les journalistes mentionnent que Pozier déclare que presque tous les artistes québécois (Audiffred 2003) et tous les auteurs québécois (Hernández 2003) sont indépendantistes; qu'il répète que la poésie joue un rôle clé dans la diffusion du Québec comme nation autonome (Anonyme 5 décembre 2003) et qu'il déclare que la Californie et la Colombie-Britannique attendent que le Québec proclame son indépendance pour faire de même (Ornelas 2003: 65).

Mais il n'y a pas que Pozier qui attire l'attention sur le nationalisme québécois. Ortiz Partida, par exemple, rapporte que Denis Vaugeois croit que le séparatisme est toujours d'actualité au Québec, en raison du fort pourcentage d'électeurs qui ont voté en sa faveur lors du référendum de 1995 (Vaugeois, cité par Ortiz Partida $1^{\text {er }}$ décembre 2003b). Dans l'article d'Ornelas, on apprend que Chassay déclare que le Québec 
constitue une fiction littéraire, car la province ne possède pas de véritable territoire (Chassay, cité par Ornelas 2003: 65). Ortiz Partida ajoute que Chassay place le débat autour de la séparation du Québec à la base de l'essai québécois actuel (Chassay, cité par Ortiz Partida $1^{\text {er }}$ décembre 2003b). Enciso confirme d'ailleurs ces dires en déclarant que les essayistes sélectionnés par Chassay «sont en majorité engagés dans des mouvements nationalistes, bien que le directeur de l'anthologie ait affirmé, au départ, avoir choisi des essais écrits au Québec, et non portant sur le Québec» (Enciso 7 décembre 2003: 10; nous traduisons) ${ }^{31}$.

À première vue, Pellerin s'applique plus, dans les médias mexicains, à insister sur les liens qui unissent le Mexique et le Québec, que sur ceux qui désunissent le Québec du Canada. Pellerin reconnaît l'influence des auteurs hispano-américains sur la nouvelle québécoise (Pellerin, cité par Ortiz Partida 2 décembre 2003c), en particulier celle des auteurs identifiés au réalisme magique (Pellerin, cité par Hernández 2003). Mais dans ses propos, la rivalité avec les Nord-Américains anglophones est palpable lorsque Pellerin mentionne, parmi les points communs entre le Mexique et le Québec, le regret que les États-Unis se soient approprié le nom d'Amérique (Mateos Vega 2003). Aussi, l'expression pueblo sin país que reprennent des journalistes mexicains (voir Enciso 29 novembre 2003: 6 et Hernández 2003:1) provient directement de la préface de Pellerin (Pellerin 2003c: 7$)^{32}$.

Audiffred résume ainsi les propos de Pozier, Chassay et Pellerin, qui auraient tous trois déclaré que, même si le ton nationaliste n'était pas évident, il serait quand même omniprésent dans la littérature québécoise actuelle:

La littérature, reconnaissent les écrivains Gilles Pellerin, Bernard Pozier et JeanFrançois Chassay, se présente comme une «forme de résistance», comme une stratégie visant à maintenir l'identité d'un peuple de 7 millions d'individus, majoritairement de langue française, et qui s'accroche à sa culture, à son drapeau, à son système politique et sa «mentalité latine». (Audiffred 29 novembre 2003; nous traduisons) ${ }^{33}$

Le recoupement des propos tenus par ces trois auteurs dans l'article suggère qu'ils ont chacun exprimé, durant la FIL, une certaine allégeance à l'idéologie nationaliste, soulignant ainsi subtilement le contenu de leur préface respective.

Certes, la préface de l'anthologie de nouvelles ne manque pas d'évoquer des préoccupations nationalistes. En plus de qualifier le Québec de pueblo sin país, Pellerin y souligne, en bas de page, la défaite du référendum de 1995 (Pellerin 2003c: 36). Cela dit, sa préface est moins didactique que celle de Pozier, et s'inscrit dans une démarche que l'on situerait davantage dans la proposition de Bourdieu, car elle fixe le champ littéraire québécois. De plus, elle impose une hiérarchie entre centre et périphérie qui rejoint la pensée d’André Lefevere (1992) et de Lawrence Venuti (1995). Elle est aussi moins politique, et plus axée sur les littératures québécoises et mexicaines. Pellerin vise d'abord à présenter les auteurs et à les inscrire dans une continuité temporelle, dans une logique historique et géographique, voire dans un combat culturel et linguistique.

La préface de Pellerin n'est traduite d'aucune autre préface publiée en français au Québec, alors que celle de Pozier, également inédite au moment de la publication de l'anthologie, sera reproduite presque littéralement, en français et en espagnol, quelques années plus tard dans le recueil intitulé 15 poètes du Québec - 15 poetas de Quebec (2008) ${ }^{34}$, également publié au Mexique. Si la préface de Pellerin semble conçue pour cette publication, elle reprend des éléments tirés de préfaces antérieures, dont 
certaines ont été rédigées pour des anthologies publiées par sa propre maison d'édition: outre l'Anthologie de la nouvelle québécoise actuelle (2003b; voir note 3), mentionnons L'atelier imaginaire (1987), Québec, des écrivains dans la ville (1995), En une ville ouverte (1990), Dix ans de nouvelles - une anthologie québécoise (1996) et, tout récemment, Vingt-cinq ans de nouvelles - une anthologie québécoise (2011) ${ }^{35,36}$. On y retrouve la passion de Pellerin pour la littérature québécoise, ainsi que son affection pour la nouvelle. Dans l'ensemble, cependant, la préface, contrairement aux préfaces des anthologies visant le public québécois, qui cherchent à présenter les auteurs dans une logique nationale plus littéraire que politique, vise ici expressément le public mexicain et présente le Québec dans un cadre où les liens avec le Mexique sont mis en relief. Pellerin passe en revue les grands moments de l'histoire québécoise, et les marqueurs nationaux et identitaires québécois sont mis en parallèle avec ceux du Mexique, ainsi que les réalités culturelles et linguistiques (débats entre français du Québec et de France, espagnol du Mexique et de l'Espagne, ou autour de la norme linguistique, de l'influence de l'anglais, etc.).

Il en est de même avec les référents historiques, économiques et géopolitiques (la proximité commune avec les États-Unis), religieuses (les liens des Mexicains et Québécois avec le catholicisme), voire démographiques (problèmes générationnels, migratoires, etc.). Le manifeste du Refus global ${ }^{37}$ est même présenté comme un pendant de mouvements mexicains de l'époque. Les grands auteurs mexicains et latinoaméricains sont également présentés dans une réflexion continentale qui les relie aux québécois, et le poète montréalais Claude Beausoleil, fort présent au Mexique, est convoqué pour appuyer cette parenté. Pellerin insiste aussi sur le rapport à la terre, au territoire, notamment à l'appropriation de la langue du terroir. Mais les termes terre, territoire et terroir, utilisés dans nombre d'articles, essais et conférences par l'éditeur dans ses réflexions sur la littérature québécoise, sont traduits de façon inégale dans la publication espagnole. Bien que nous n'ayons pas eu accès au texte original, il ne fait pas de doute, étant donné la précision avec laquelle Pellerin utilise toujours ces termes, qu'ils ont été traduits ici avec un souci moindre quant à la connotation du référent. Ainsi le terme terruño, dont on peut estimer sans trop de risque de se tromper qu'il traduit le terme terroir, évoque en espagnol une odeur de campagne qui s'éloigne considérablement du sens que Pellerin accorde à ce terme - le terroir de Pellerin exprime davantage un lien émotif fort avec le territoire, voire une forme d'appropriation locale de la langue, que quelque champêtre état d'âme.

Cette question de l'appropriation de la langue du terroir revient sous différentes facettes dans la préface de l'anthologie de nouvelles. Il n'est pas innocent par exemple que soit cité le cas du terme blé d'Inde, utilisé au Québec plus fréquemment que le terme mä̈s, que Pellerin n'oublie d'ailleurs pas de relier à son origine arawak (Pellerin 2003c: 20; voir note 32). L'importance des termes liés à la forêt est également relevée, cette forêt, précise Pellerin, omniprésente dans l'imaginaire québécois, et partant dans la littérature québécoise, étant un autre exemple de la place du terroir dans notre littérature, un terroir ici encore qui n'a guère à voir avec quelque fragrance bucolique. Or, cette forêt, que Pellerin associe à une masse qui écrase littéralement, mais aussi métaphysiquement, les Québécois, est traduite par bosque (Pellerin 2003c: 12, 20, 21, 23, 24, 26, 29; voir note 32), ce qui évoque difficilement les étendues d'épinettes noires qui donnent tout son sens à la métaphore souhaitée par le préfacier. Mais il est vrai que cette forêt n'a guère à voir non plus avec l'image de la maison de pierres inondée 
de soleil et de verdure tendre de la page couverture. À croire finalement qu'il y a peut-être eu un léger décalage entre le souhait de l'anthologiste/préfacier et celui de l'éditeur mexicain.

Enfin, la place de la nouvelle dans la littérature québécoise constitue une autre occasion de rapprochement entre le Québec et le Mexique, l'influence de la nouvelle latino-américaine sur la québécoise étant évidente pour Pellerin ${ }^{38}$, et même déterminante eu égard à son désir d'inclure notre littérature dans les grandes littératures d'Amérique. Parmi les caractéristiques rapprochant la nouvelle québécoise et latinoaméricaine, Pellerin cite: la construction des recueils dans une logique narrative dépassant la juxtaposition des textes, le travail sur la langue, les modifications de la trame, l'importance du fantastique, la fugacité et l'anonymat des personnages, l'ironie, la solitude, la déshumanisation de la grande ville, etc. Le fait que la nouvelle n'ait jamais atteint au Québec le même niveau de popularité qu'au Mexique ou ailleurs en Amérique latine est laissé dans l'ombre: un silence qui ne surprend guère, car il y a là une véritable différence entre le Québec et ses voisins du sud ${ }^{39}$.

Si la préface de Pellerin n’a jamais été reproduite depuis, celle de Chassay est parue deux fois la même année (et non pas «en différé» comme celle de Pozier): en espagnol dans El hilo de la memoria (Chassay 2003c; voir note 26) et en français dans Anthologie de l'essai au Québec depuis la Révolution tranquille (Chassay 2003d; voir note 26). Cette double parution n'est cependant pas connue des lecteurs mexicains, puisque la version française est absente de l'édition mexicaine de l'anthologie. En revanche, il est précisé dans l'édition québécoise que l'anthologie «est proposée simultanément au public québécois et au public mexicain puisqu’elle est également publiée en espagnol, à Mexico, par le Fondo de Cultura Económica» (Chassay 2003d: 15). Toujours dans la même note, on précise que «le choix des textes est le même. Seule cette préface présente quelques différences, notamment dans le but de contextualiser l'histoire québécoise récente pour les Mexicains» (Chassay 2003d: 15, nous soulignons). Voyons en quoi consistent exactement les différences entre les préfaces en espagnol et en français, et si toutes visent vraiment à expliciter le «contexte historique» québécois aux lecteurs mexicains.

Dès le premier paragraphe de la version en espagnol, on trouve un élément de contextualisation absent du texte français, soit une explication du choix des années 1960 comme première période représentée dans la sélection d'essais, qui «correspond à une période de transformations capitales survenues dans la société québécoise, et dont les premiers soubresauts ont pu être ressentis dans les années qui ont suivi la Deuxième Guerre mondiale. Cette évolution sociale a eu des répercussions majeures sur le plan culturel.» (Chassay 2003c: 7; nous traduisons) ${ }^{40}$. En français, Chassay justifie son choix différemment, en insistant davantage sur les changements littéraires et en nommant déjà clairement la «Révolution tranquille» par son nom (ce qu'il ne fait que plusieurs lignes plus tard en espagnol), expliquant que ce "point de départ chronologique a un sens réel sur le plan culturel, ne serait-ce qu'à cause du développement de l'institution littéraire»(Chassay 2003d: 7). Plus loin, dans le texte en espagnol, une incise explique que le joual est la langue populaire de Montréal («lengua popular de Montreal»; Chassey 2003c: 8), une information qui aurait effectivement été superflue pour un lectorat québécois. Un ajout du même type se retrouve là encore dans le texte en espagnol, cette fois au sujet de la colonisation du territoire qui allait devenir le Québec: «au début de la colonisation, le Canada s'appelait la 
Nouvelle France, jusqu'à la conquête anglaise de la Guerre de 7 ans, entre 1756 et $1763 »$ (Chassey 2003c: 14) ${ }^{41}$. Par contre, un autre passage de la préface espagnole que l'on ne retrouve pas en français n'a rien de vraiment contextualisant; il s'agit seulement d'une remarque sur la nouveauté du contenu de l'anthologie pour un public mexicain: "On peut penser que chaque auteur sera, pour le public mexicain, une nouvelle découverte» (Chassay 2003c: 12) ${ }^{42}$.

Cela dit, il est intéressant de constater que le texte français compte lui aussi des précisions contextuelles dont les lecteurs mexicains n’ont pas bénéficié: ainsi, Chassay écrit que la poésie est encore « un des genres de prédilection» des éditions de l'Hexagone (Chassay 2003d: 7) ; une dizaine de lignes portent sur les collections très dynamiques consacrées au genre de l'essai qui auraient joué «un rôle déterminant dans les années 1960» (Chassay 2003d: 8); à la fin du même passage, Chassay explique que dans les années 1970, «un nombre de plus en plus grand de maisons d'édition sentent le besoin d'intéresser leurs lecteurs» à l'essai (Chassay 2003d: 8); enfin, une parenthèse explique que «l'essai n'est pas contraint par des frontières disciplinaires, ce qui explique bien les difficultés à circonscrire les siennes»(Chassay 2003d: 12). Autant d'informations absentes de la préface en espagnol.

\section{Les tables des matières: quelle information?}

La consultation des tables des matières permet de faire quelques constatations relatives au choix des langues de publication et à la présence des femmes parmi les auteurs retenus.

\subsection{Le bilinguisme: une nécessité imposée par la poésie?}

Les trois tables des matières ne donnent pas la même quantité d'information au sujet du contenu des anthologies. Ainsi, la table des matières de l'anthologie de poésie, Latinos del norte, est très exhaustive: elle compte huit pages et présente toutes les sections de la préface, puis le nom de chacun des poètes retenus, suivi des années de naissance et, le cas échéant de décès, de ceux-ci, puis une liste complète des titres de poèmes inclus, en français et en espagnol. La table des matières de l'anthologie d'essais, El hilo de la memoria, est quant à elle unilingue, beaucoup plus courte (trois pages) et construite différemment. Seul le titre de la préface («Introducción») est indiqué, puis les essais sont regroupés en sept thèmes (I. Europa, América; II. Historia; III. Política; IV. Cultura y sociedad; V. Feminismo; VI. Lengua; VII. Escribir, leer, pintar), sous lesquels on trouve le titre espagnol de l'essai, suivi du nom de son auteur. Enfin, la table des matières de l'anthologie de nouvelles ¿Un continente a la deriva? est beaucoup plus sommaire et tient sur une seule page. Les noms de chacun des 17 auteurs inclus, suivis du titre espagnol de la nouvelle traduite, sont présentés l'un après l'autre. Il est difficile de déterminer ce qui a pu motiver l'ordre de présentation: les noms ne sont ni en ordre alphabétique ni, semble-t-t-il, en ordre chronologique strict. Contrairement à l'anthologie de poèmes, les tables de l'anthologie de nouvelles et de l'anthologie d'essais ne sont pas bilingues, ce qui semble indiquer qu'aux yeux des éditeurs, la poésie mérite davantage d'attention sur le plan de la forme et que ses lecteurs sont plus soucieux d'exactitude, de formalisme, de musicalité linguistique. 


\subsection{La littérature québécoise, une "terre des hommes"?}

Si une chose saute aux yeux à la lecture des tables des matières des trois anthologies, c'est une présence des femmes notablement plus faible que celle des hommes. Dans El hilo de la memoria, les femmes représentent exactement le quart des essayistes sélectionnés ( 6 sur 24 ; voir annexe 3 ) et ne signent qu'un peu plus du quart des textes présentés (11 sur 43 , soit presque $26 \%$ ), dont 3 se concentrent dans la section traitant du féminisme. La préface contribue à cette identification entre femmes et féminisme, car c'est dans cette section que, selon Chassay, le lecteur aura accès à une réflexion depuis une perspective fondamentalement féminine (Chassay 2003c: 16). Chassay explique qu'il a retenu des auteurs établis comme essayistes à partir des années 1960 et dont l'ensemble de l'œuvre est reconnue par leurs pairs et par le grand public (Chassay 2003c: 12). La faible proportion de femmes porte à croire que l'essai est, au Québec, un genre à prédominance masculine. La consultation de la base de données en ligne de l'Infocentre littéraire des écrivains québécois (ÎLE ${ }^{43}$ ) renforce cette hypothèse, puisque, en 2010, seulement $27 \%$ des essayistes répertoriés (95 sur 353) sont des femmes.

Dans ¿Un continente a la deriva?, la proportion de femmes est un peu plus élevée, presque le tiers des nouvellistes traduits (6 sur 17, soit $35 \%$; voir annexe 3 ). C'est un peu en dessous de la proportion de femmes (48\%) chez les nouvellistes recensés par l'ÎLE (138 sur 285, en 2010; voir note 43). Par ailleurs, Pellerin, dans sa préface, accorde moins de place aux femmes qu'aux hommes qu'il a choisis pour son anthologie. En effet, il consacre environ deux pages à Robert Lalonde (Pellerin 2003c: 25-26; voir note 32) et environ une demi-page respectivement à Gaëtan Brulotte (Pellerin 2003c: 31), Jean-Paul Baumier (Pellerin 2003c: 31-32), Louis Jolicoeur (Pellerin 2003c: 36-37), ainsi qu'à lui-même (Pellerin 2003c: 37). En revanche, il n'accorde que quelques lignes à Monique Proulx (Pellerin 2003c: 29), auxquelles s'ajoutent quelques mentions éparses qui finissent par équivaloir à une demi-page (Pellerin 2003c: 11, 33, 36). Il ne fait que nommer au passage Aude (Pellerin 2003c: 10, 27, 33), Diane-Monique Daviau (Pellerin 2003c: 11, 28, 33), Marie Josée Thériault (Pellerin 2003c: 11 et 34) et Suzanne Jacob (Pellerin 2003: 28). Le nom de Sylvie Massicotte n'apparaît qu'en notes de bas de page (Pellerin 2003c: 10, 33).

Enfin, dans Latinos del norte, presque le tiers des poètes traduits sont des femmes ( 5 sur 15, soit $30 \%$ ). Ce chiffre est un peu inférieur à la proportion de femmes poètes répertoriées par l'ÎLE, soit environ $36 \%$ (169 sur 464; en 2010, voir note 43). Sur les 42 poètes que Pozier mentionne dans sa préface, 18 sont des femmes (42\%), mais 10 d'entre elles sont énumérées en rafale, en lien avec le mouvement féministe des années 1970 (voir les auteures repérées par une étoile dans l'annexe 4). En fait, seules Anne Hébert et Nicole Brossard, qui font d'ailleurs partie des poètes traduits dans Latinos del norte, semblent ressortir du lot. Dans la préface, Hébert est nommée 3 fois: aux côtés des «grands aînés » Saint-Denys Garneau et Grandbois (Pozier 2003b : 10; voir note 27); parmi les poètes réédités à la fin des années 1990 ou au début des années 2000 (Pozier 2003b: 14); puis de nouveau avec Garneau et Grandbois dans la conclusion de la préface, qui présente le contenu de l'ouvrage (Pozier 2003b: 16). Quant à Brossard, elle est citée parmi les féministes des années 1970 (Pozier 2003b: 13), puis plus loin comme codirectrice de l'Anthologie de la poésie des femmes au Québec ${ }^{44}$ (Pozier 2003b: 13); son nom refait surface avec les poètes qui ont récemment atteint 
leur «maturité poétique» (Pozier 2003b : 14); puis il réapparaît en conclusion (Pozier 2003b : 16).

Il est intéressant de remarquer que la place des femmes poètes dans Latinos del norte est analogue à celle que Pozier leur a accordée dans l'anthologie Poetas de Quebec (Blouin, Carvajal, et al. 1996) ${ }^{45}$, où l'on retrouvait 16 femmes sur les 56 poètes traduits $(28,5 \%)$. Néanmoins, le contraste est frappant avec une autre anthologie traduite parue en 2003 à l'occasion de la FIL, elle aussi dirigée par Pozier (2003c ${ }^{46}$, intitulée Aquí y ahora: 35 poetas de Quebec a partir de 1960. Dans cette dernière, on compte 16 femmes parmi les 35 poètes sélectionnés, tous nés après 1960, soit une présence féminine de $45 \%$. Un lecteur mexicain qui achèterait les deux anthologies parues en 2003 (voir la comparaison en annexe 4) pourrait donc avoir l'impression qu'il y a, chez les poètes nés après 1960, une plus grande proportion de femmes que chez ceux des générations précédentes.

Si chacune des trois anthologies semble réunir une proportion de femmes représentative de la réalité «démographique» de chacun de ces genres spécifiques au Québec, il reste qu'elles accordent de façon générale une place plutôt réduite aux femmes: sur l'ensemble des auteurs traduits réunis dans les trois ouvrages, à peine plus du quart sont des femmes (15 sur 53, soit $28 \%$ ), et cela en tenant compte du fait que certains auteurs sont publiés dans plus d'un ouvrage ${ }^{47}$. Pourtant, environ la moitié des membres de l'UNEQ sont des femmes ${ }^{48}$. De plus, la base de données de l'ÎLE révèle que $42 \%$ des auteurs répertoriés, tous genres confondus, sont des femmes (537 sur 1279; voir note 43). Bien que les sites de l'UNEQ et de l'ÎLE ne réunissent pas nécessairement tous les auteurs québécois, et que la liste de genres littéraires auxquels les auteurs sont identifiés ne soit pas toujours exhaustive, ces données fournissent un point de comparaison valable, une forte proportion des auteurs québécois y étant tout de même représentés.

\section{Le contenu des anthologies : du passé poétique au présent narratif}

La sélection des textes des anthologies a été faite dans les trois cas par le directeur littéraire d'une maison d'édition québécoise: Bernard Pozier dans celui de l'anthologie de poèmes, Gilles Pellerin dans le cas de l'anthologie de nouvelles, et JeanFrançois Chassay pour l'anthologie d'essais. Ce sont donc des Québécois, non des Mexicains, qui sont à l'origine du choix des auteurs et des textes traduits. Cette réalité contraste avec les propos de Luise von Flotow sur la littérature canadienne traduite en Allemagne, selon qui, "[in] the case of two "B" nations like Canada and Germany, [...] it is the importing culture that makes the decisions in regard to selection» (von Flotow 2007: 198; nous soulignons). Ici, c'est plutôt la culture exportatrice qui a décidé ce qu'elle voulait bien faire traduire. Cela dit, nous avons vu que seule l'anthologie de poèmes a été produite spécifiquement pour un public mexicain, les anthologies de nouvelles et d'essais ayant aussi été présentées la même année en français à un public québécois.

Chose sûre, lorsqu'on feuillette en parallèle le contenu des trois anthologies du FCE, on constate qu'elles se suivent chronologiquement. Latinos del norte offre une sélection moins contemporaine que les deux autres ouvrages. D'abord, le premier tiers de ses auteurs (5 sur 15) sont nés avant 1920, le deuxième, autour de 1930, et le troisième tiers, entre 1943 et 1949. C'est donc dire qu'en 2003, au moment de la publi- 
cation, 7 des 15 poètes étaient décédés, tandis que les 8 autres avaient déjà entre 55 et 75 ans. En quatrième de couverture, on lit que le survol proposé par Latinos del norte présente le fruit des "principales transformations survenues dans les années trente et les années soixante» (Pozier 2003a: $4^{\text {e }}$ de couverture) ${ }^{49}$, ce qui laisse à entendre que les textes plus récents de Nicole Brossard, Normand de Bellefeuille, Yolande Villemaire, Claude Beausoleil et Lucien Francoeur sont, eux aussi, un produit des idéologies des années 1960. Dans les faits, cependant, certains poèmes traduits n'ont pas été produits dans cette perspective. Les poèmes de Brossard sont tirés du recueil Au présent des veines paru en $1999^{50}$, qui contient des œuvres rédigées entre 1981 et 1999. Encore plus récents, les textes de Bellefeuille et de Francoeur sont tirés de recueils parus en $2001^{51}$ et ceux de Beausoleil, d'un ouvrage de 2002 (Les passions extérieures) $)^{52}$.

Les médias mexicains ont repris au moins deux fois (Lecturas del Fondo de Cultura Económica et La Jornada $)^{53}$ le texte expliquant en quatrième de couverture que l'anthologie présente une poésie ayant marqué les temps forts de l'histoire littéraire québécoise des années 1930 aux années 1960. Pour sa part, Sonia Sierra écrit que l'anthologie de poèmes renferme diverses générations de poètes du $\mathrm{xx}^{\mathrm{e}}$ siècle (Sierra 2 décembre 2003a; 2 décembre 2003b; voir annexe 2$)^{54}$, sans se prononcer sur leur représentativité. Pour Iván Castañeda, moins impartial, Pozier a réuni « un échantillon de la poésie québécoise la plus représentative du $\mathrm{xx}^{\mathrm{e}}$ siècle»(Castañeda 2007: 32$)^{55}$. De même, Mariana Islas précise que Pozier s'est soucié d'offrir aux lecteurs mexicains «un panorama equilibrado» de la poésie québécoise (Islas $1^{\text {er }}$ décembre 2003) ${ }^{56}$. En revanche, le quotidien El Universal semble moins positif lorsqu'il affirme que Pozier agglutine une quinzaine de poètes dans son anthologie (Anonyme 29 novembre 2003: 4 ; voir annexe 2). Quant au journaliste JFC du journal Mural, il est clairement d'avis que la sélection de Pozier constitue une étude plutôt «archéologique» et il déplore le fait que le lecteur mexicain n'ait pas accès à l'œuvre de plus jeunes poètes (JFC décembre 2003: 14; voir annexe 2). En fait, les choix chronologiques de Pozier pourraient s'expliquer par le parallèle établi entre son anthologie et une autre, mexicaine, Antología de la poesía mexicana moderna, dirigée par Jorge Cuesta, parue pour la première fois en $1928^{57}$. Sa réédition, avec les traductions en français par Émile et Nicole Martel, est coéditée par le FCE et les Écrits des Forges à l'occasion de la FIL ${ }^{58}$. Selon Sierra, ces deux anthologies «sont des ponts qui permettent le partage, de part et d'autre, de la tradition du genre poétique» (Sierra 2 décembre 2003a; 2 décembre 2003 b; voir annexe 2 ; nous traduisons $)^{59}$. Notons par ailleurs que Pozier a inclus, dans Latinos del norte, des poètes qui ne sont pas associés aux Écrits des Forges. Ainsi, les textes de Saint-Denys Garneau, Alain Grandbois, Anne Hébert, Roland Giguère, Paul-Marie Lapointe, et Gaston Miron proviennent d'autres maisons d'édition.

Si l'on analyse l'anthologie d'essais, El hilo de la memoria, d'un point de vue chronologique, Chassay rapporte que les essayistes qu'il a choisis sont tous nés entre 1920 et 1958 (Chassay 2003c: 12). Cependant, on remarque à la lecture des notices biographiques que la moitié d'entre eux (12 sur 24) sont nés après 1940, et que 19 vivaient toujours au moment de la publication du livre. En ce qui concerne les textes originaux eux-mêmes, une majorité d'entre eux (25 sur 42) ont été rédigés, ou publiés pour la première fois, au cours des années 1960 (3 textes), 1970 (9 textes) ou 1980 (13 textes). Toutefois, bon nombre d'entre eux sont assez récents, le tiers (12 textes) datant des années 1990, et 5 autres, des années 2000. Le fait d'ordonner 
les essais de façon chronologique permet de sustenter le commentaire d'Ortiz Partida (2 décembre 2003; voir annexe 2), selon qui l'anthologie fournirait un portrait de la vie au Québec durant les quarante dernières années. Le quotidien El Universal va dans ce sens, jugeant que les textes traduits offrent au lecteur mexicain une idée de la pensée québécoise au sujet d'une variété de thèmes tels que la politique, le féminisme, la langue, l'art, la culture, la société, l'histoire, etc. (Anonyme 29 novembre 2003). Selon Hernández, les thèmes traités par les essayistes ont même une portée internationale: "ils abordent des thèmes qui résonnent partout dans le monde, comme l'identité, les femmes ou la mort» (Hernández 2 décembre 2003: 1; nous traduisons; voir annexe 2$)^{60}$.

Nous avons vu plus haut (voir section 4) que Chassay affirme à plusieurs reprises, dans sa préface (Chassay 2003c) mais aussi dans de nombreuses entrevues rapportées dans la presse, qu'il a voulu réunir des textes qui proviennent du Québec, plutôt que des textes qui traitent nécessairement du Québec. Selon Enciso, cependant, la majorité des essais traduits sont teintés d'une idéologie nationaliste (Enciso 7 décembre 2003 ; voir section 4 et note 31 ) et ce, malgré ce qu'a pu en dire le compilateur, peu importe le thème traité. Il faut dire que Chassay aurait déclaré en entrevue que la forme de l'essai actuel repose sur le débat identitaire et indépendantiste (Chassay, cité par Ortiz Partida $1^{\text {er }}$ décembre 2003b). À cette occasion, Denis Vaugeois aurait renchéri en expliquant que le genre de l'essai a été à l'origine de nombreux changements sociaux au Québec et aurait donné comme exemple l'essai Option Québec de René Lévesque (Vaugeois, cité par Ortiz Partida $1^{\text {er }}$ décembre 2003b). Il est donc possible que ces propos, présentés publiquement lors de la FIL et repris dans la presse écrite quelques jours avant la publication du compte rendu d'Enciso, aient influencé la lecture du critique.

Enfin, l'anthologie de nouvelles, ¿ Un continente a la deriva?, dirigée par Pellerin, se distingue des deux autres par une sélection d'œuvres nettement plus contemporaines. À part Roland Bourneuf, né en 1934, et Jean Pierre Girard, né en 1961, tous les auteurs sélectionnés sont nés dans les années 1940 (8 sur 17) ou 1950 (8 sur 17), sont vivants au moment de la publication, et font partie de la génération que l'on associe aux pionniers de la nouvelle contemporaine québécoise. De plus, presque tous les textes originaux peuvent être considérés comme récents: 15 nouvelles sur 17 sont tirées de recueils parus au cours des années 1990 (13 sur 17) ou 2000 (2 sur 17). Les auteurs ont, pour la plupart (12 sur 17), publié aux éditions de L'instant même. Cela est explicable: d'une part, le responsable de la publication est l'un des fondateurs et aujourd'hui directeur littéraire de la maison d'édition, d'autre part, celle-ci se spécialise dans la nouvelle. On constate cependant un désir d'ouvrir la sélection à des auteurs d'autres maisons, dont Boréal et Québec-Amérique.

Les auteurs sélectionnés par Pellerin sont, dans l'ensemble, des nouvellistes consacrés. Dans la préface et les notices biographiques, c'est d'ailleurs sur leur œuvre en tant qu'auteur de nouvelles que l'accent est mis. Ainsi, si la carrière de romancier de Robert Lalonde est amplement relevée, ainsi que son travail d'acteur, on passe toutefois sous silence sa fréquentation de la poésie. On laisse également dans l'ombre le fait que Marie José Thériault est la fille d'Yves Thériault, dont il est toutefois question ailleurs dans la préface. La place d'un Hugues Corriveau dans le milieu de la poésie québécoise, et de l'essai, n’est pas relevée non plus. En bref, il est évident que l'accent est mis sur la nouvelle contemporaine québécoise, au risque de donner 
l'impression qu'elle occupe une place peut-être plus grande que cela n'est le cas dans le panorama littéraire québécois. À cet égard, Enciso, journaliste déjà cité plus haut, reste lucide. Sachant que l'histoire de la nouvelle québécoise est encore jeune, il écrit: «réaliser une compilation historique des nouvelles au sein de littérature québécoise a peu d'intérêt: le risque de ne pas accéder à un matériel suffisant est important» (Enciso 29 novembre 2003: 6; voir annexe 2; nous traduisons) ${ }^{61}$. Enciso estime cependant que la sélection de Pellerin fournit un tour d'horizon représentatif de la vitalité de la scène littéraire québécoise actuelle, et se réjouit que les nouvelles sélectionnées s'éloignent d'un régionalisme que l'on aurait pu craindre pour adopter un caractère universel ${ }^{62}$. Le critique littéraire de Mural, JFC, va dans le même sens, jugeant que Pellerin a su réunir un éventail des voix narratives les plus représentatives du Québec actuel, regrettant quand même un peu que le compilateur ait inclus une de ses propres nouvelles, aussi excellente soit-elle (JFC décembre 2003: 14).

Quoi qu'il en soit, pour un lecteur mexicain qui se procurerait les trois livres, il ressortirait de ces choix chronologiques que les temps forts de la poésie semblent associés au passé littéraire québécois, que la nouvelle littéraire paraît plus proche de son présent (voire de son avenir), et que l'essai semble faire le pont entre le Québec de la Révolution tranquille et celui du $\mathrm{XxI}^{\mathrm{e}}$ siècle. Cette impression se concrétise d'ailleurs à la lecture des préfaces de Pellerin (2003c; voir note 32) et de Chassay (2003c; voir note 26). Pellerin accorde une bonne page aux poètes canadiens-français du XIX ${ }^{\mathrm{e}}$ siècle, s'attardant en particulier à Octave Crémazie et à Louis Fréchette (Pellerin 2003c: 15-16). Il mentionne aussi l'importance du poète Gaston Miron durant les années 1960 (Pellerin 2003c: 24), mais c’est la nouvelle littéraire qui émerge comme genre proprement contemporain. Comme on peut le lire en quatrième de couverture, c'est elle qui «a insufflé sa vitalité à la littérature du Québec» («ha infundido vitalidad a la literatura de Quebec» (Pellerin 2003a: 4e de couverture; voir note 3 ; nous traduisons). Quant à Chassay, il souligne dans sa préface la place prépondérante de la poésie après la Deuxième Guerre mondiale et dans les années 1950, et il précise que plusieurs considèrent la poésie comme l'un des moteurs de la Révolution tranquille (Chassay 2003c: 8 ; voir note 26). Il écrit également que, depuis les années 1990, l'essai a perdu de sa popularité au profit de travaux de nature scientifique, aujourd'hui de plus en plus valorisés dans le monde universitaire (Chassay 2003c: 8-9), ce qui pourrait expliquer pourquoi la majorité des essais sélectionnés ont été rédigés avant 1990.

\section{Rayonnement des anthologies et des auteurs traduits : place aux poètes!}

Les textes des poètes inclus dans Latinos del norte semblent jouir d'une meilleure diffusion dans la presse mexicaine que ceux des auteurs des deux autres anthologies. Par exemple, ce sont les poètes qui prennent le plus de place en novembre 2003 dans le numéro spécial de la Gaceta del Fondo de Cultura Económica consacré à la promotion des ouvrages publiés à l'occasion de la FIL (Saísar Guerrero 2003) ${ }^{63}$. Des 27 pages de textes que comporte la revue, près de six sont consacrées à l'anthologie de poésie, contre quatre, à l'anthologie d'essais, et un peu plus de trois, à l'anthologie de nouvelles. Alors que l'on y publie des extraits des préfaces de Pellerin et de Chassay, celle de Pozier y est reprise intégralement. Par ailleurs, on y reproduit une seule nouvelle, Nadette y otros nombres (de Louis Jolicœur) et un seul essai, Monsieur Melville (de 
Victor Lévy-Beaulieu), mais pas moins de trois poèmes: En la plaza pública de Gaston Miron; La primavera de Quebec de Gatien Lapointe et Una amiga de Montreal de Claude Beausoleil. Enfin, la version espagnole du poème Angustia Story de Lucien Francoeur est reproduite le 2 décembre 2003 dans le quotidien El Financiero. Le 5 décembre, le même journal diffuse quatre autres textes tirés de l'anthologie de Pozier: Jaula de pájaro de Saint-Denys Garneau; Extraña captura d’Anne Hébert; La mano del verdugo termina por pudrirse de Roland Giguère; et La primavera de Quebec de Gatien Lapointe. Ces inclusions des poèmes traduits par Marco Antonio Campos dans la revue du FCE ainsi que dans El Financiero ont sans doute contribué à en appuyer la diffusion auprès du grand public.

Même au sein des anthologies de Pellerin et Chassay, les poètes québécois sont omniprésents, bien que leur présence ne soit pas toujours déclarée ouvertement. En effet, trois des nouvellistes sélectionnés sont aussi des poètes: Suzanne Jacob, Robert Lalonde et Hughes Corriveau. Or, comme il a été indiqué plus haut, Pellerin ne mentionne pas que ceux-ci ont écrit de la poésie. Ainsi, l'on apprend dans la notice biographique de Jacob que celle-ci a eu une carrière de chanteuse (Pellerin 2003a: 41) et, dans celle de Lalonde, que celui-ci s'est aussi démarqué comme romancier et dramaturge (Pellerin 2003a: 65), mais aucun des cas, on ne parle de leur œuvre poétique. Pourtant, un recueil des poèmes de Lalonde, paru en 2002, circule déjà au Mexique en version espagnole ${ }^{64}$. En fait, seule la notice biographique de Corriveau révèle que son œuvre prolifique compte non seulement des nouvelles, mais de la poésie, des essais et des romans (Pellerin 2003a: 217).

En entrevue au Mexique, Chassay déclare que la nouvelle littéraire est selon lui plus proche du genre poétique que romanesque (Chassay, cité dans Ortiz Partida 2 décembre 2003). Dans sa préface, il établit même un parallèle entre la poésie, la philosophie et l'essai, expliquant que bon nombre des essayistes québécois «sont également - et, parfois, surtout - nouvellistes, poètes ou philosophes» (Chassay 2003c: 9; voir note 26; nous traduisons) ${ }^{65}$. D'ailleurs, cinq des 24 auteurs réunis dans El hilo de la memoria ne sont pas classés parmi les essayistes dans la base de données de l'ÎLE: Daniel Jacques et Jean Morisset ne sont pas répertoriés du tout, tandis que Nicole Brossard est associée à la poésie et au roman; Madeleine Gagnon à la poésie, au roman et au récit; Gaston Miron à la poésie. À la lecture des notices biographiques des essayistes, on constate que près de la moitié d'entre eux (11 sur 24 en 2010) sont poètes. Or, s'il est vrai, comme l'explique Chassay en préface, qu'un même texte puisse tenir à la fois de l'essai, de la théorie ou de l'analyse littéraire, l'extrait de L'homme rapaillé de Miron, qui apparaît dans la dernière section du livre, tient plus du poème que de l'essai proprement dit.

Il faut relever que l'on nomme souvent les traducteurs de l'anthologie de poésie dans la presse mexicaine, ce qui donne, là encore, une visibilité particulière au genre. Parmi les 17 articles des dossiers de la FIL traitant de l'une ou l'autre des anthologies, six nomment les traducteurs de Latinos del norte ${ }^{66}$, Marco Antonio Campos et Mónica Mansour, qualifiés de «traducteurs magnifiques» (JFC décembre 2003: 14; voir annexe 2). Le contraste est frappant avec les traducteurs des deux autres anthologies. Laura López Morales et Margarita Montero, qui ont transposé ¿Un continente a la deriva?, ne sont citées qu'une fois en passant, et moins pour leur traduction que parce que López Morales a animé une table ronde sur la nouvelle littéraire (Ortiz Partida 2 décembre 2003: 4). Quant à Glenn Gallardo, traducteur 
de El hilo de la memoria, il n'est nommé par aucun journaliste du dossier de presse de la FIL. Même Enciso, qui fait pourtant la recension de chacun des deux ouvrages (Enciso 29 novembre 2003a: 6; 7 décembre 2003b: 10), ne fait aucune mention de leurs traducteurs.

Pourtant, dans l'anthologie de poésie, le traducteur de chaque poème est clairement nommé. Les essais, quant à eux, ont été traduits par la même personne. En revanche, on ne sait pas avec certitude qui des deux traductrices de l'anthologie de nouvelles signe chaque version, l'invisibilité du traducteur étant ici nettement plus manifeste que dans le cas des poèmes et des essais. Sur la scène littéraire, où les journalistes ont tendance à éclipser les noms des traducteurs, le contraste entre l'invisibilité de Gallardo, la presque invisibilité de Morales et Montero, et la visibilité de Campos et Mansour incite à penser que la renommée des traducteurs de l'anthologie de poèmes avait le potentiel de contribuer à faire vendre l'ouvrage.

\section{Conclusion}

Au terme de ce survol des paratextes, trois tendances se dessinent: l'évocation de l'histoire (ou du terroir) québécoise; l'insistance sur l'appartenance du Québec à une culture latino-américaine; et le regret d'un pays qui aurait pu (ou pourrait encore) être. D'abord, l'effet d'ensemble créé par les tableaux de Garneau en couverture évoque plus le passé que le présent du Québec. Si La liseuse aux tons chauds est facilement assimilable à la latino-américanité du titre de l'anthologie, La vieille chaufferie cadre moins bien avec le continent à la dérive de Pellerin. Pourtant, la lecture des préfaces donne presque l'impression que les titres des anthologies de poésie et de nouvelles ont été intervertis. En effet, Pozier mentionne à plusieurs reprises l'absence de pays du Québec et le fait que Mexicains et Québécois sont «sans continent», tandis que Pellerin souligne sans relâche la latinité intrinsèque des nouvellistes québécois. Un commentaire de Pellerin (2003c: 10; voir note 32) porte à croire que l'anthologie de nouvelles aurait peut-être pu porter le titre Venus du Nord, qui évoque franchement Latinos del norte. De fait, les parallèles entre Québécois et Mexicains ne manquent pas dans les trois préfaces, ni les allusions, directes ou indirectes, au nationalisme québécois, à la fois dans les commentaires des compilateurs rapportés par la presse écrite et dans les trois préfaces, en particulier celle de Pozier.

Sur le plan du choix d'auteurs, c'est l'anthologie de poésie qui semble vraiment représenter le passé littéraire, l'anthologie de nouvellistes se concentrant plutôt sur le présent et l'avenir de la littérature québécoise. Quant à l'anthologie d'essais, son titre fait référence à la mémoire, évoquant le passé, mais son contenu paratextuel, depuis l'Arrière-cour de Westmount de la jaquette jusqu'à la préface et la table des matières, met plutôt l'accent sur le caractère à la fois moderne et urbain de l'essai québécois. En outre, ce sont surtout les écrits d'hommes québécois que les compilateurs ont retenus dans les anthologies du FCE. Pourtant, la proportion réelle de femmes écrivaines est beaucoup plus élevée au Québec que ce que les trois anthologies ne pourraient laisser croire. Enfin, la poésie semble l'avoir emporté sur les autres genres en matière de visibilité dans la façon dont la littérature québécoise a été présentée par le FCE et par les médias mexicains, ainsi que dans les préfaces et dans la sélection des auteurs des trois anthologies, et ce, au risque de donner à penser que le Québec est avant tout une terre de poètes. Reste maintenant à savoir 
comment la littérature québécoise a été présentée aux lecteurs mexicains au moyen de la traduction des textes eux-mêmes, ce qui fera sans nul doute l'objet d'une future recherche.

\section{NOTES}

* La recherche a été subventionnée par le Fonds institutionnel de développement de la recherche et de la création (FIRC) de l'Université du Québec en Outaouais (UQO) et le Fonds de recherche du Québec sur la santé et la culture (FRQSC). Nous remercions la contribution de nos assistants Magali Cloutier Provencher, Barbara Menjívar et Marco Suárez, ainsi que l'équipe éditoriale de Meta.

1. Morissette, Brigitte (8 décembre 1996): Le livre québécois attaque la forteresse latino-américaine. La Presse. B5.

2. Le Fondo de Cultura Económica (FCE) est la plus importante maison d'édition au Mexique. Consulté le 21 décembre 2011, <http://www.fondodeculturaeconomica.com>.

3. Pellerin, Gilles dir. (2003a): ¿Un continente a la deriva? Antología de narradores de Quebec. (Traduit par Laura López Morales et Margarita Montero) Collection Tierra Firma. Mexico: Fondo de Cultura Económica.

Pellerin, Gilles dir. (2003b): Anthologie de la nouvelle québécoise actuelle. Québec: L’instant même.

4. PozIer, Bernard, dir. (2003a): Latinos del norte. Antología de poesía de Quebec. (Traduit par Marco Antonio Campos et Mónica Mansour) Collection Tierra Firma. Mexico/Québec: Fondo de Cultura Económica/Écrits des Forges.

5. Chassay, Jean-François, dir. (2003a): El hilo de la memoria. Antología de ensayo de Quebec. (Traduit par Glenn Gallardo) Collection Tierra Firma. Mexico: Fondo de Cultura Económica. Chassay, Jean-François, dir. (2003b): Anthologie de l'essai au Québec depuis la Révolution tranquille. Montréal: Éditions du Boréal.

6. FONDO DE CULTURA ECONÓMICA (29 septembre 2010): Historia del FCE (Última actualización por Emilio Alanis). Secretaría de Educación Pública, Estados-Unidos Mexicanos. Consulté le 21 décembre 2011, <http://www.fondodeculturaeconomica.com/Institucional/HistoriaFCE.asp >.

7. FONDO DE CULTURA ECONÓMICA (s. d.): Cómo publicar en el FCE. Consulté le 21 décembre 2011, <http://www.fondodeculturaeconomica.com/editorial/Publicar/Default.aspx $>$.

8. BUREAU DES ÉVÉNEMENTS DU QUÉBEC (2003): ; Voilà Québec en México! Revue de presse (Cédéroms). Référencé dans Bibliothèque et Archives Canada. Consulté le 14 mars 2014, <http:// collectionscanada.gc.ca/pam_archives/index.php?fuseaction=genitem.displayItem\&lang=fra\&rec _nbr=4361528>.

9. Lecturas del Fondo de Cultura Económica, XVII Feria Internacional del Libro de Guadalajara 2003. Presencia del FCE Numero especial, 11/2003, p. 7-14.

10. "Québec, Fondo de Cultura Económica" dans La Jornada: 27 et dans La Reforma, 28 novembre 2003: 35 .

11. S'il est vrai que les trois ouvrages sont le fruit d'une coédition, seule l'anthologie de poésie (Latinos del norte) porte, sur la page couverture, le logo de la maison québécoise, Écrits des forges. Les deux copyrights sont d'ailleurs indiqués à l'intérieur du livre. L'anthologie de nouvelles ( $\dot{i}$ Un continente a la deriva ?), quant à elle, ne porte que le copyright du FCE. Enfin, l'anthologie d'essais (El hilo de la memoria) porte le copyright du FCE dans le monde hispanique, mais celui des Éditions du Boréal pour le reste du monde. Par ailleurs, les essais de Pierre Vadeboncoeur et de Gaston Miron portent aussi le copyright de Typo, et ceux de François Dumont, celui d'Hurtubise HMH.

12. Gascon, France (2011): L'Univers de Saint-Denys Garneau - Le peintre, le critique. Montréal: Éditions du Boréal.

13. Il déclarait, en octobre 1929: «[...] mon intention est de me vouer à l’art: à la poésie et à la peinture» (Saint-Denys Garneau 1929, cité dans Huot 2004: 220).

14. Saint-Denys Garneau, Hector de (s. d.): La liseuse. Huile sur toile, $29,2 \times 20,3 \mathrm{~cm}$. Collection Bibliothèque Anne-Hébert, Municipalité de Sainte-Catherine-de-la-Jacques-Cartier. Consulté le 14 mars 2014, <http://www.saintdenysgarneau.com/infoPhoto.htm>.

15. Piste située entre Pont-Rouge à Sainte-Catherine-de-la-Jacques-Cartier, à l'ouest de la ville de Québec. Voir PistesCyclables.ca, consulté le 14 mars 2014, <http://pistescyclables.ca/Quebec/ Liseuse.htm>. 
«Parcours dans la nature lié à l'œuvre de l'écrivain et qui explore des aspects de son œuvre là même où elle s'est élaborée.» Le Sentier de la Liseuse, VirtualMuseum.ca. Consulté le 14 mars 2014, $<$ http://www.museevirtuel-virtualmuseum.ca/GetMuseumEvent.do?lang=en\&chinCode=guaesl \&eventCode $=2>$.

16. Charest, Rémy (22 octobre 1993a): La musique actuelle fête Saint-Denys Garneau. Le Devoir. B8. Charest, Rémy (9 octobre 1993b): Saint-Denys Garneau: le peintre, le poète et sa famille. Le Devoir. D1.

LEPAGE, Jocelyne (25 novembre 1995): Saint-Denys Garneau peintre, à la Bibliothèque nationale. La Presse. D9.

Montpetit, Caroline (10 mars 2001): Saint-Denys Garneau en peinture. Le Devoir. D3.

17. On peut ici songer à Calliope, muse grecque de la poésie. Voir l'article qui lui est consacré dans l'Encyclopoedia Britannica.

EnCyClopaedia Britannica (2014): Calliope. Encyclopoedia Britannica Online Academic Edition. Consulté le 2 avril 2014, <http://www.britannica.com/EBchecked/topic/89997/Calliope>.

18. Voir Audiffred (29 novembre 2003; annexe 1) et Petrowski (6 décembre 2003).

Petrowski, Nathalie (6 décembre 2003): Feria Internacional del Libro: Ce n'est qu'un au revoir amigos. La Presse. 5.

19. [Saint-Denys Garneau, Hector de] (s. d. [1930]): La vieille chaufferie. Aquarelle, $14,2 \mathrm{~cm} \times$ $11,5 \mathrm{~cm}$. Collection Giselle Huot, Montréal. Citée dans Huot (2004: 270).

20. Saint-Denys Garneau, Hector de (s. d.): Arrière-cours Montréal. Huile sur toile, $30,6 \mathrm{~cm} \times$ 21,5 cm. Collection Ville de Westmount. Consulté le 14 mars 2014, <http://www.saintdenys garneau.com/expo/?attachment_id=23>.

21. Apostolska, Aline (14 mars 2011): Gaston Bellemare: le bilan anorexique de visibilité du livre québécois. BSC News Magazine. Consulté le 13 avril 2011, <http://www.bscnews.fr/201103141442/ Les-Grandes-Interviews/gaston-bellemare-le-bilan-anorexique-de-visibilite-du-livre-quebecois. html>.

22. Campos, Marco Antonio (2010): Une approche personnelle de la poésie québécoise. L’Unique Journal de l'UNEQ. 12(3):3.

23. Landry y déclare: «creemos que somos los latinos del norte y es un factor muy positivo para nosotros ver a los latinos del sur que aumentan su desarrollo, se organizan [...]» (Landry, cité dans Carreño Figueras 13 mai 1999).

Carreño Figueras, José (13 mai 1999): Landry Moneda Común. Servicio Universal de Noticias (SUN).

24. SAunders, Doug (20 mai 2002): Quebec expanding missions in the U.S. The Globe and Mail. A4.

25. Y aurait-il lieu de voir dans le titre de Chassay une référence indirecte à la fameuse devise «Je me souviens» gravée au-dessus de la porte principale de l'Assemblée nationale et reproduite sur les plaques d'immatriculation québécoises, et dont la signification reste plutôt ambigüe pour bon nombre de Québécois? Au sujet de la devise, voir l'explication sur le site de Justice Québec.

GOUVERNEMENT DU QUÉBEC (Dernière mise à jour: 14 janvier 2008): La devise du Québec - Je me souviens. Justice Québec. Consulté le 22 avril 2014, <http://www.drapeau.gouv.qc.ca/devise/ devise.html>.

26. Chassay, Jean-François (2003c): Introducción. In: Jean-François Chassay, dir. El hilo de la memoria - Antología de ensayo de Quebec. (Traduit par Glenn GALlardo) Collection Tierea Firma. México : Fondo de Cultura Económica, 7-17.

Chassay, Jean-François (2003d): Introduction. In: Jean-François Chassay. Anthologie de l'essai au Québec depuis la Révolution tranquille. Montréal: Éditions du Boréal, 7-15.

27. Pozier, Bernard (2003b) : Prólogo. In: Bernard Pozier, dir. Latinos del norte. Antología de poesía de Quebec. (Traduit par Marco Antonio Campos et Mónica Mansour) Collection Tierra Firma. Mexico/Québec: Fondo de Cultura Económica/Écrits des Forges, 7-17.

28. «la casi totalidad de los intelectuales y artistas quebequenses son independentistas, separatistas o en favor de la autonomía» (Pozier 2003b: 8).

29. «sin continente, porque el nombre lo han usurpado los estadunidenses, sin poder, por estar bajo la férula de los ricos anglófonos, mantenido en la ignorancia por su propio gobierno y privado de deleites por las prohibiciones de su clero» (Pozier 2003b: 10).

30. «poco a poco asumida política y económicamente» (Pozier 2003b : 11).

31. «los 23 [sic] autores que aportan textos a esta colección [...] incurren, de modo mayoritario, en tratamientos nacionalistas aun cuando el compilador de la obra haya pretendido, inicialmente, hacer una selección del ensayo de y no sobre Quebec» (Enciso 7 décembre 2003: 10) 
32. Pellerin, Gilles (2003c) : Prólogo. In: Gilles Pellerin, dir. ¿Un continente a la deriva? Antología de narradores de Quebec. (Traduit par Laura López Morales et Margarita Montero) Collection Tierra Firma. Mexico: Fondo de Cultura Económica, 7-40.

Pellerin, Gilles (2003d): Préface - Le temps inquiet. In: Gilles Pellerin, dir.: Anthologie de la nouvelle québécoise actuelle. Québec: L’instant même, 7-21.

33. «La literatura, reconocen los escritores Gilles Pellerin, Bernard Pozier y Jean-Francois Chassay, se yergue como una "forma de resistencia", como estrategia para mantener la identidad de un pueblo con 7 millones de habitantes y una población mayoritariamente francófona que se aferra a su cultura, su bandera, su sistema político y su "mentalidad latina" [...]» (Audiffred 29 novembre 2003).

34. PozIer, Bernard, dir. (2008): 15 poetas de Quebec - 15 poètes du Québec. Colección terredades/ Collection Poésie internationale. Tlaquepaque/Trois-Rivières: Mantis Editores/Écrits des Forges.

35. Rouquet, Guy, dir. (1987): L'atelier imaginaire. Québec: L'instant même.

Pellerin, Gilles, dir. (1990): En une ville ouverte. Québec: L’instant même - Atelier du Gué. Pellerin, Gilles, dir. (1995): Québec, des écrivains dans la ville. Québec: L'instant même - Musée du Québec.

Pellerin, Gilles, dir. (1996): Dix ans de nouvelles - une anthologie québécoise. Québec: L'instant même.

Mottet, Philippe et Pellerin, Gilles, dir. (2011): Vingt-cinq ans de nouvelles - Une anthologie québécoise. Québec: L’instant même.

36. Outre les ouvrages publiés aux éditions L'instant même, il faut aussi signaler le recueil de nouvelles argentines et québécoises intitulé Rencontres / Encuentros - Escritores y artistas de Argentina y Quebec / Écrivains et artistes d'Argentine et du Québec (1989), également préfacé par Gilles Pellerin. [s. n.] (1989): Rencontres / Encuentros - Escritores y artistas de Argentina y Quebec / Écrivains et artistes d'Argentine et du Québec (Traduit par Louis Jolicoeur et Cecilia PonTe) Montréal: Les Éditions Sans Nom.

37. Borduas, Paul-Émile (1948): Refus global. Montréal: Éditions Mythra-Mythe. Consulté le 14 mars 2014, <http://fr.wikisource.org/wiki/fr:Refus_global ?oldid=3721483>.

38. Voir, entre autres, son article intitulé Influence argentine sur la nouvelle québécoise (Pellerin 2010).

39. Pellerin n'ignore cependant pas le statut précaire de la nouvelle au Québec, il l'a d'ailleurs fort clairement documenté dans son essai intitulé Nous aurions un petit genre (Pellerin 1997).

40. «[...] corresponde a un periodo de transformaciones capitales que ocurrieron en la sociedad quebequense, y cuyos primeros sobresaltos pudieron sentirse en los años posteriores a la segunda Guerra Mundial. Esta evolución social tendrá repercusiones muy importantes en el terreno cultural» (Chassay 2003c: 7).

41. «[...] a principios de la colonización, Canadá se llamó la Nueva Francia, hasta la conquista inglesa durante la guerra de Siete Años entre 1756 y $1763 »$ (Chassay 2003c: 14).

42. «Para el público mexicano, es posible pensar que cada autor sea un nuevo hallazgo (Chassay 2003c: 12).

43. L'TLE. L'infocentre littéraire des écrivains québécois. Consulté le 20 mai 2010, <http://www.litterature.org/recherche>.

44. Brossard, Nicole et Girouard, Lisette (1991): Anthologie de la poésie des femmes au Québec. Montréal: Éditions du Remue-ménage. Réimprimé en 1992 et réédité en 2003 chez le même éditeur.

45. Blouin, Louise, Carvajal, Juan, Pozier, Bernard, et al., dir. (1996): Poetas de Quebec: breve antologia. Mexico: DF UNAM.

46. PozIer, Bernard, dir. (2003c): Aquí y ahora: 35 poetas de Quebec a partir de 1960. (Traduit par Mónica López et Eduardo Padilla) Mexico: Filodecaballos Editores.

47. Nicole Brossard, Gaston Miron et Suzanne Jacob apparaissent dans deux des trois anthologies.

48. En date du 5 avril 2011, le répertoire en ligne de l'UNEQ comptait 731 femmes sur un total de 1442 membres. Union des écrivaines et des écrivains québécois (UNEQ). Consulté le 5 avril 2011, $<$ http://www.uneq.qc.ca $>$.

49. «las principales transformaciones ocurridas en los años treinta y los años sesenta» (Pozier 2003a: $4^{\mathrm{e}}$ de couverture).

50. Brossard, Nicole (1999): Au présent des veines. Trois-Rivières/Luxembourg/La Réunion: Écrits des Forges/Grand Océan.

51. De Bellefeuille, Normand (2001): Un visage pour commencer. Trois-Rivières/Marseille: Écrits des Forges/Autre temps. 
Francoeur, Lucien (2001): Express pour l'Éden. Trois-Rivières/Pantin: Écrits des Forges/Le temps des Cerises.

52. Beausoleil, Claude (2002): Les passions extérieures. Trois-Rivières/ Marseille: Écrits des Forges/ Autre temps.

53. Anonyme (novembre 2003): Quebec, los Latinos del norte. Lecturas del Fondo de Cultura Económica - Numéro especial. 10.

Anonyme (21 novembre 2003) : Latinos del Norte. Antología de poesía de Quebec. La Jornada. 6.

54. Le même texte est paru le même jour dans deux journaux.

55. «una muestra de lo más representativo de la poesía quebequense del siglo XX» (Castañeda 2007 : 32).

Castañeda, Iván (2007): Latinos del norte (Antología de poesía de Quebec); Bernard Pozier. Norte/Sur. 4(17):32.

56. Islas, Mariana ( ${ }^{\text {er }}$ décembre 2003): Oferta FCE panoramas literarios de Quebec. Mural.

57. Cuesta, Jorge (1928): Antología de la poesía mexicana moderna. Mexico: Contemporaneos.

58. Cuesta, Jorge (1928/2003): Anthologie de la poésie mexicaine moderne = Antología de la poesía mexicana moderna. Trois-Rivières/Mexico : Écrits des Forges/FCE.

59. «extienden puentes para que aquí y allá sea compartida la tradición de este género [poesía] (Sierra 2 décembre 2003a; voir annexe 2).

60. «abordan temas que en cualquier parte del mundo tienen resonancia, como la identidad, las mujeres o la muerte» (Hernández 2 décembre 2003: 1).

61. «Poco útil resulta elaborar una recopilación histórica de las novelas cortas en las letras quebequenses; el riesgo de no hallar material suficiente para hacerlo es alto» (Enciso 29 novembre 2003: 6).

62. Enciso commente le nationalisme particulier et complet («peculiar y complejo») du Québec, mais conclut, en lisant l'anthologie, qu'il est un fait de la nature ("hecho de la naturaleza») plus que le fruit d'une idéologie inculquée (Enciso 29 novembre 2003: 6).

63. Saísar Guerrero, Consuelo, dir. (novembre 2003): Latinos del Norte: Cultura y literatura de Quebec. (Núme especial) La Gaceta del Fondo de Cultura Económica, 395. Publié par le Fondo de cultura economica. (Consulté le 21 décembre 2011, <http://www.fondodeculturaeconomica.com/ subdirectorios_site/libros_electronicos/Gacetas/nov_2003/index.html>.

64. Lalonde, Robert (2002): Bahía crepitante. (Traduit par Silvia Pratt) Mexico: Mantis editores.

65. «[..] son igualmente - y a veces principalmente - novelistas, poetas o filósofos» (Chassay 2003c: 9).

66. Voir annexe 2: Anonyme (5 décembre 2003: 61), Cárdenas (23 novembre 2003a: 71), JFC (décembre 2003: 14), Ortiz Partida ( $1^{\text {er }}$ décembre 2003a), Sierra (2 décembre 2003a: $2 ; 2$ décembre 200b: 28), ainsi que Anonyme (21 novembre 2003: 6; voir note 53).

\section{RÉFÉRENCES}

Armony, Victor (2002): Des Latins du Nord? L'identité culturelle québécoise dans le contexte panaméricain. Recherches sociographiques. 43(1):19-48.

Audet, René (2002): La fiction à l'essai. In: Alexandre GEFEn et René Audet, dir. Frontières de la fiction. Collection Fabula. Montréal/Bordeaux: Éditions Nota bene/Presses universitaires de Bordeaux, 133-157.

BeAusoleil, Claude (2004): Los Latinos del Norte - La traduction à la XVII ${ }^{\mathrm{e}}$ Feria Internacional del Libro de Guadalajara. Lettres québécoises. 114:52.

Bellemare-Page, Stéphanie (2004): Un bilan commercial très positif pour plusieurs éditeurs québécois. Livres d'ici. 29(6):14-16.

Bourdieu, Pierre (2002): Les conditions sociales de la circulation internationale des idées. Actes de la recherche en sciences sociales. 145:3-8.

Everett, Jane et Marcotte, Sophie (2010): De l'anthologie. Voix et Images. 35(2):7-15.

Genette, Gérard (1987): Seuils. Paris: Éditions du Seuil.

HÉBERT, François (1998): La peinture de Saint-Denys Garneau. Liberté. 40(4):4-24.

Huot, Giselle (2004): L'aventure artistique du peintre De Saint-Denys Garneau. Mens / Revue d'histoire intellectuelle de l'Amérique française. IV(2):211-271. 
Lefevere, André (1992): Translation, Rewriting \& the Manipulation of Literary Fame. London/ New York: Routledge.

Pellerin, Gilles (1997): Nous aurions un petit genre - Publier des nouvelles. Québec: L'Instant même.

Pellerin, Gilles (2010): Influence argentine sur la nouvelle québécoise. Nuit blanche, magazine littéraire. 120:67-73.

Pratt, Silvia (2007): Traduire les auteurs québécois au Mexique: un maillon entre deux cultures. In: Louis Jolicoeve, dir. Traduction et enjeux identitaires dans le contexte des Amériques. Québec: Presses de l'Université Laval-CEFAN, 37-52.

Venuti, Lawrence (1995): The Translator's Invisibility - A History of translation. London/New York: Routledge.

von Flotow, Luise (2007): Revealing the "soul of which nation?" Translated literature as cultural diplomacy. In: Paul St-Pierre et Prafulla C. Kar, dir. In Translation - Reflections, Refractions, Transformations. Amsterdam/Philadelphie: John Benjamins, 187-200.

Yuste FríAs, José (2010): Au seuil de la traduction: la paratraduction. In: Antonius Bernardus Maria NaAjukns, dir. Event or Incident. Événement ou Incident. On the Role of Translation in the Dynamics of Cultural Exchange. Bern: Peter Lang, 287-316.

\section{ANNEXES}

Annexe 1 - Auteurs québécois invités à la FIL de Guadalajara de 2003, selon le dossier de presse

\begin{tabular}{|l|l|l|l|}
\hline José Acquelin & Chrystine Brouillet & Daniel Jacques & Michel Noël \\
Marie-Célie Agnant & Jean-François Chassay & Louis Jolicœur & Stanley Péan \\
Claude Beausoleil & Gil Courtemanche & D. Kimm & Gilles Pellerin \\
Lise Bissonnette & Marie-Danielle Croteau & Sergio Kokis & Bernard Pozier \\
Neil Bissoondath & Daniel Danis & Robert Lalonde & Rober Racine \\
Louise Bombardier & Dominique Demers & Suzanne Lebeau & Élise Turcotte \\
Gérard Bouchard & Trevor Ferguson & Viateur Lefrançois & Denis Vaugeois \\
Michel Marc Bouchard & Bertrand Gauthier & Geneviève Letarte & Gilles Vigneault \\
Serge Bouchard & Marie-Louise Gay & Guy Marchamps & \\
Nicole Brossard & David Homel & Rita Mestokosho & \\
\hline
\end{tabular}


Annexe 2 - Textes tirés de la revue de presse ; Voilà Québec en México! (voir note 8)

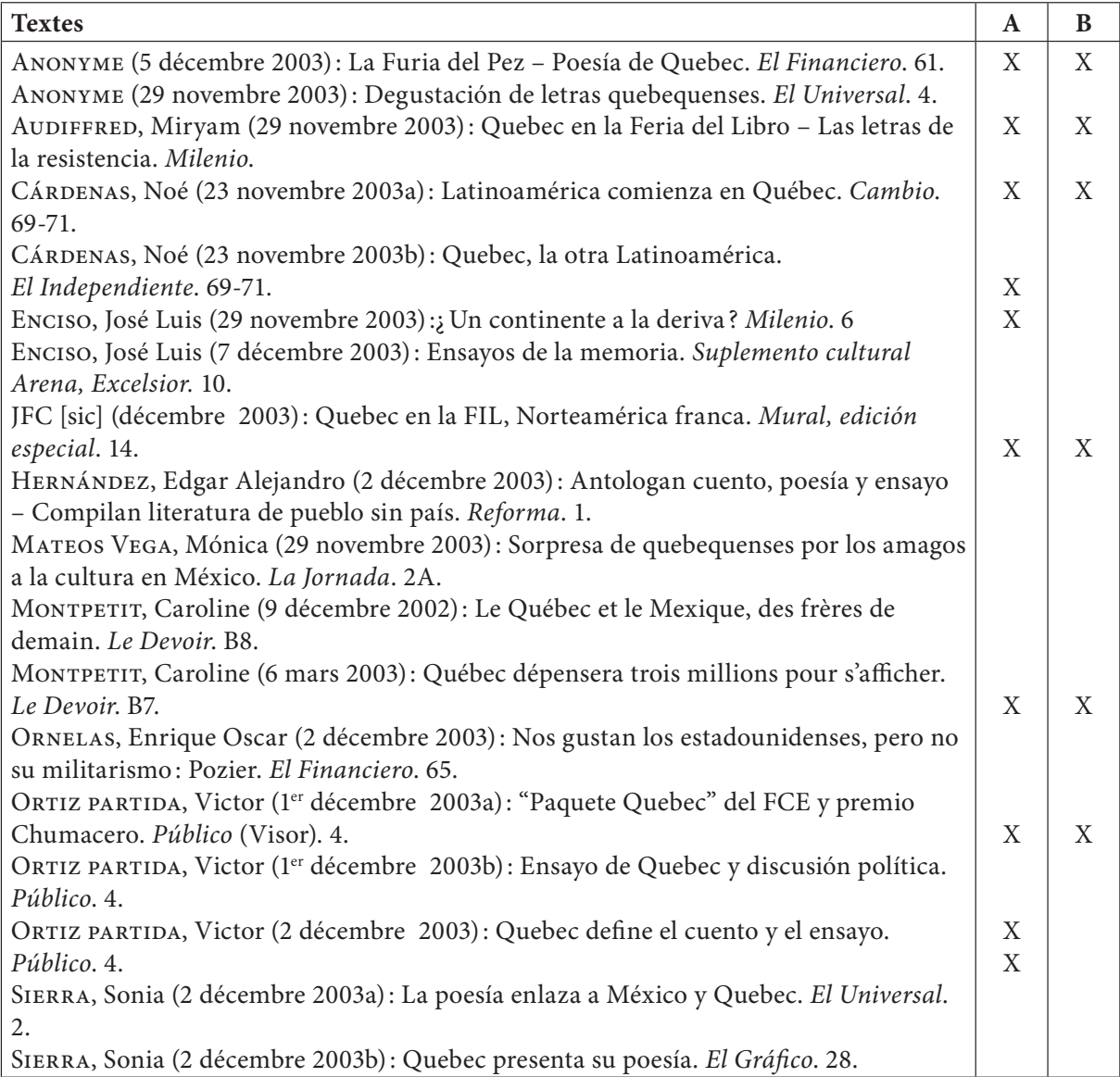

A : articles faisant référence à l'indépendance ou au nationalisme québécois;

B: articles dans lesquels sont cités les propos de Pozier ou des extraits de sa préface. 
Annexe 3 - La présence des femmes dans les anthologies d'essai (El hilo de la memoria) et de nouvelles (¿ Un continente a la deriva?)

\begin{tabular}{|c|c|c|c|}
\hline \multicolumn{2}{|c|}{$\begin{array}{c}\text { El hilo de la memoria } \\
\text { (Chassay 2003a; voir note } 3 \text { ) }\end{array}$} & \multicolumn{2}{|c|}{$\begin{array}{l}\text { ¿Un continente a la deriva? } \\
\text { (Pellerin } 2003 \text {; voir note } 4)\end{array}$} \\
\hline $\begin{array}{l}\text { Auteures } \\
(6)\end{array}$ & $\begin{array}{c}\text { Auteurs } \\
(18)\end{array}$ & $\begin{array}{l}\text { Auteures } \\
(6)\end{array}$ & $\begin{array}{l}\text { Auteurs } \\
(11)\end{array}$ \\
\hline $\begin{array}{l}\text { Suzanne Jacob } \\
\text { Régine Jobin } \\
\text { Lise Bissonnette } \\
\text { Nicole Brossard } \\
\text { Madeleine Gagnon } \\
\text { Suzanne Lamy }\end{array}$ & $\begin{array}{l}\text { Victor-Lévy Beaulieu } \\
\text { Gilles Marcotte } \\
\text { Jean Morisset } \\
\text { Pierre Nepveu } \\
\text { François Ricard } \\
\text { André Belleau } \\
\text { Daniel Jacques } \\
\text { René Lapierre } \\
\text { Paul Chamberland } \\
\text { Mordecai Richler } \\
\text { Laurent-Michel Vacher } \\
\text { Fernand Dumont } \\
\text { Pierre Vadeboncœur } \\
\text { Jean Larose } \\
\text { Jean Marcel } \\
\text { Jacques Brault } \\
\text { André Brochu } \\
\text { Gaston Miron }\end{array}$ & \begin{tabular}{|l|} 
Monique Proulx \\
Aude \\
Diane-Monique Daviau \\
Marie José Thériault \\
Suzanne Jacob \\
Sylvie Massicotte
\end{tabular} & $\begin{array}{l}\text { Robert Lalonde } \\
\text { Gaëtan Brulotte } \\
\text { Jean-Paul Beaumier } \\
\text { Louis Jolicoeur } \\
\text { Gilles Pellerin } \\
\text { Michel Dufour } \\
\text { Jean Pierre Girard } \\
\text { Roland Bourneuf } \\
\text { Hans-Jürgen Greif } \\
\text { Hugues Corriveau } \\
\text { Bertrand Bergeron }\end{array}$ \\
\hline
\end{tabular}

Annexe 4 - La présence des femmes dans deux anthologies de poésie de Pozier parues en 2003 au Mexique

\begin{tabular}{|c|c|c|c|c|}
\hline \multicolumn{3}{|c|}{$\begin{array}{c}\text { Latinos del norte. Antología de poesía de Quebec } \\
\text { (Pozier 2003a; voir note 4) }\end{array}$} & \multicolumn{2}{|c|}{$\begin{array}{c}\text { Aquí y ahora: } 35 \text { poetas de Quebec a } \\
\text { partir de } 1960 \\
\text { (Pozier 2003c; voir note 46) }\end{array}$} \\
\hline $\begin{array}{l}\text { Auteures citées } \\
\text { dans la préface } \\
\text { (18) }\end{array}$ & $\begin{array}{l}\text { Auteures } \\
(5)\end{array}$ & $\begin{array}{c}\text { Auteurs } \\
(10)\end{array}$ & $\begin{array}{l}\text { Auteures } \\
(16)\end{array}$ & $\begin{array}{c}\text { Auteurs } \\
(19)\end{array}$ \\
\hline $\begin{array}{l}\text { Anne Hébert } \\
\text { Nicole Brossard } \\
\text { France Théoret } \\
\text { Denise Boucher } \\
\text { Madeleine } \\
\text { Gagnon } \\
\text { Louise Cotnoir } \\
\text { Madeleine } \\
\text { Ouelette- } \\
\text { Michalska } \\
\text { Louise Dupré } \\
\text { Germaine } \\
\text { Beaulieu } \\
\text { Louise Desjardins } \\
\text { Carole David } \\
\text { Thérèse Renaud } \\
\text { Rina Lasnier } \\
\text { Lisette Girouard } \\
\text { Yolande } \\
\text { Villemaire } \\
\text { Denise Desautels }\end{array}$ & $\begin{array}{l}\text { Anne Hébert } \\
\text { Rina Lasnier } \\
\text { Thérèse Renaud } \\
\text { Nicole Brossard } \\
\text { Yolande } \\
\text { Villemaire }\end{array}$ & $\begin{array}{l}\text { Hector de Saint- } \\
\text { Denys-Garneau } \\
\text { Alain Grandbois } \\
\text { Alphonse Piché } \\
\text { Roland Giguère } \\
\text { Paul-Marie } \\
\text { Lapointe } \\
\text { Gaston Miron } \\
\text { Gatien Lapointe } \\
\text { Normand de } \\
\text { Bellefeuille } \\
\text { Claude Beausoleil } \\
\text { Lucien Francoeur }\end{array}$ & $\begin{array}{l}\text { Hélène Monette } \\
\text { Nancy R. Lange } \\
\text { Martine Audet } \\
\text { Louise } \\
\text { Beauchamp } \\
\text { Pascale Des } \\
\text { Rosiers } \\
\text { Denise Brassard } \\
\text { Aline Poulin } \\
\text { Anne Peyrouse } \\
\text { Chantale } \\
\text { Lévesque } \\
\text { Béatrice } \\
\text { Migneault } \\
\text { Cynthia Girard } \\
\text { Isabelle Forest } \\
\text { Annie Perrault } \\
\text { Anick Arsenault } \\
\text { Julie Dorval } \\
\text { Rosalie Lessard }\end{array}$ & \begin{tabular}{|l} 
Jean Perron \\
Francis Catalano \\
Mario Cholette \\
Fernand Durepos \\
Michel Pleau \\
Jean-François \\
$\quad$ Poupart \\
Jean Boisvert \\
Stéphane Despatie \\
Jean Duval \\
Martin Pouliot \\
Tony Tremblay \\
Louis Cornellier \\
Éric Roberge \\
Frédéric Gary \\
Comeau \\
Jean-Sébastien \\
$\quad$ Huot \\
Jean-Éric Riopel \\
Carl Lacharité \\
Patrick Léveillé \\
Alexandre Trudel
\end{tabular} \\
\hline
\end{tabular}

* Citations en lien avec les mouvements féministes des années 1970. 